\title{
Spectral characteristics of moist birch leaves and synthetic materials: experimental studies and evaluation of models
}

\author{
Alexander Mikkelsen $\odot$ * and Gorm K. Selj \\ Norwegian Defence Research Establishment, Kjeller, Norway
}

\begin{abstract}
The optical depth of biomaterials and natural backgrounds varies with their environment and incoming light properties. Deciduous trees, for instance, have canopy thicknesses that vary significantly from one place to another. Leaves constituting canopies absorb most of the light in the visible region, while transmitting a large ratio of the incoming near-infrared light. Such variations in the spectral properties of biomaterials pose complicated challenges for developing camouflage material and precise land area mapping by remote sensors. We aim to address these challenges by investigating multi-layered birch leaves, a biomaterial abundant throughout Northern Europe. We measured and compared the reflectance, transmission, and absorptance of moist birch leaves between 250 and $2500 \mathrm{~nm}$ and compared their values with the spectral properties of three different synthetic materials. We found that the spectral properties of the synthetic materials matched those of the birch leaf at certain wavelengths and material thicknesses. The results highlight the importance of choosing appropriate material thickness when designing camouflage and having knowledge of the thickness variation of the biomaterial constituting the background. Furthermore, the results are of relevance for wavelength-dependent detections based on spectral anomalies of artificial objects when recorded through vegetation layers. Moreover, we tested the spectral data of the materials against an earlier published extinction model. With few exceptions, the model fit the spectral data well and could be used to estimate the spectral properties of the materials as a function of their thickness. We also compared the accuracy of the extinction model with two other models and discussed their value and practicality. Our findings will be valuable for the development of camouflage materials as well as advanced multi-layered fabric technology and remote sensing applications. (C) The Authors. Published by SPIE under a Creative Commons Attribution 4.0 International License. Distribution or reproduction of this work in whole or in part requires full attribution of the original publication, including its DOI. [DOI: 10.1117/1.OE.60 .11.117102]
\end{abstract}

Keywords: optical properties; reflectance; transmission; absorption; imaging; infrared imaging.

Paper 20210375 received Apr. 7, 2021; accepted for publication Oct. 12, 2021; published online Nov. 9, 2021.

\section{Introduction}

Effective concealment in natural backgrounds is an important capability in the animal kingdom and warfare alike. The main purpose of camouflage is to blend in with the environment and attract a minimum of attention from any given observer or sensor. Effective camouflage is normally achieved through matching with the characteristic background, resulting in minimized contrasts between camouflage materials and natural constituents in the background of relevance. Spectrally, this means reducing material contrast to the background by capturing the background reflectance and transmittance properties, ensuring that the camouflage material and the background reflects and transmits light similarly over any relevant wavelength range. ${ }^{1-5}$ Spatial matching with the background is normally ensured through frequency and orientation optimization. ${ }^{5-10}$

In the years to come, electro-optical sensors are expected to become smaller, more widespread, cost-effective, and capable. As sensors and analysis of sensor data are increasingly more sophisticated and easily accessible (including from drones), remote sensing capabilities are expected to be of enhanced relevance to military units. Concealing a person or object is

*Address all correspondence to Alexander Mikkelsen, alexander.mikkelsen@ffi.no 
increasingly difficult and demands multi-spectral as well as spectrally optimized camouflage material to provide protection against several sensor threats. ${ }^{3,11-13}$

Camouflage nets typically consist of fabrics arranged in multiple layers when viewed from the perspective of incident light rays and may be comprised of a background material covered with leaf-like patches. Textiles for uniforms are typically made of single layer, and their effective optical thickness is a result of a compromise of several needs, rather than just the desired spectral reflectance properties. In comparison, multi-layered camouflage fabrics provide several favorable properties such as suppression of thermal contrast outliers, ${ }^{14}$ improved thermal comfort, ${ }^{15}$ electromagnetic signature control, ${ }^{16}$ increased shadowing effects, and preventing revealing effects such as unnaturally flat surfaces in natural cluttered backgrounds.

According to known literature, several studies have been carried out assessing camouflage net effectiveness ${ }^{11,12,17-20}$ and camouflage textile effectiveness ${ }^{3,5,6,8,21-24}$ but mainly considering spectral reflectance (or pattern) background matching. Little has been reported on spectral transmission properties of camouflage materials as a function of both (i) the number of layers in a stack and (ii) wavelength. However, some studies have reported on spectral transmission measurements for textile fibers ${ }^{25-28}$ and spectral reflectance as a function of the number of stacked layers. ${ }^{17}$ Still, in natural backgrounds consisting of many thin biomaterial structures, such as deciduous leaves, transmission effects have been found to significantly affect measured spectral reflectance, all depending on wavelengths considered. ${ }^{29,30}$

In particular, it has been shown that incident light in the visible, near-infrared (NIR), and short-wave infrared radiation (SWIR) penetrates thin biomaterial structures, such as in deciduous canopies, to different depths because of wavelength-dependent transmittance. ${ }^{29,30}$ It is our hypothesis that light in the NIR and SWIR wavelengths when measured by any remote sensor is also expected to contain reflected light from any underlying structures such as soil, concealed objects, or other parts of the background. On the other hand, light detected in the visible spectrum (and in ranges at 1450 and $1900 \mathrm{~nm}$ dominated by water absorption) is expected to be dominated by reflected light from the vegetation. Due to the optical behavior of thin biomaterials, there will most likely be characteristic differences in optical properties when synthetic concealment materials are compared to natural materials. These differences must be better understood with additional measurements and theoretical models. Several models aiming to better understand the electromagnetic interactions with natural leaves have been developed over decades, ranging from simple plate models ${ }^{30-32}$ to advanced ray tracing models. ${ }^{33,34}$ However, none of these models directly compares the spectral properties of stacked natural leaf-structures with layers of synthetic camouflage material (e.g., leaf-like fabrics for camouflage nets or textiles for uniforms).

There are two main purposes with this study: (1) to compare measurements of biological and synthetic materials and (2) to explore the applicability of theoretical models that can be used in a predicative sense. The first point relies on experimental measurements of reflectance, transmittance, and absorption properties for stacked layers of (i) natural birch leaves and (ii) synthetic camouflage fabrics. The second point aims to better understand the feasibility of mathematical models, describing optical properties of stacks of thin semitransparent material, that have not earlier, to the best of the authors' knowledge, been applied to data from experiments carried out on materials such as leaves and thin synthetic materials. The aim is to increase the knowledge on thin-layered structures, and how their numbers of layers affect their characteristic optical properties. In addition, the models tested are thought to be useful in predicting inherent optical properties of materials where direct measurements can sometimes be very difficult or cost-ineffective.

We studied brown birch leaves (Betula pendula) due to seasonal availability and because such leaves are abundant throughout Northern Europe in several months of every year. The study also adds to the existing knowledge on the construction of synthetic materials that can capture the complex optical properties of thin biomaterial structures. Furthermore, mathematical models, as earlier suggested in the literature, ${ }^{25,30}$ were used to fit experimental data yielding a deeper, basic knowledge on light transmittance in thin, semitransparent structures, and perforated layers. We have modified the model suggested by Lillesaeter ${ }^{30}$ to also account for multiple reflections among the layers and compared these two models to the extinction model ${ }^{25}$ where also light absorptance is accounted for. The results found that in this study are thought to be valuable to any further improvements and developments in advanced (and multi-layered) camouflage 
products. The results are also expected to be of interest for remote sensing purposes where vegetated land areas, such as given by tree canopies are mapped throughout a full year cycle as well as plant water content mapping. ${ }^{4,35-39}$

In this study, we report on the following: 2 to 3 layers of brown birch leaves and synthetic camouflage material were sufficient to prevent the background from influencing the measured reflectance in the visual band. In the NIR wavelength range, however, leaves were found to transmit light until a thickness of seven or eight layers, whereas the synthetic materials were less transparent, resulting in a reduced number of layers needed to hinder background contributions (perforated: 2 to 4 , textile: 5 to 6 ). The three theoretical models used were adequate in their ability to fit experimental data, albeit with important differences. The extinction model was found to be the better model among the three (best fit to the experimental data) by accounting for absorption effects, in addition to multiple scattering and characteristics of the background.

\section{Experimental Setup and Method}

\subsection{Spectral Measurements}

A PerkinElmer spectrophotometer (Lambda 750S UV/VIS/NIR) was used to measure the reflectance and transmission of birch leaves and textile samples (Fig. 1) for incoming light of wavelengths between 250 and $2500 \mathrm{~nm}$. We used a deuterium lamp (Part. No. L6022728, PerkinElmer) between 250 and $319.2 \mathrm{~nm}$ and a tungsten lamp (Part. No. B0114620, PerkinElmer) between 319.2 and $2500 \mathrm{~nm}$ as illuminants. The relative spectral power of the illuminants is presented
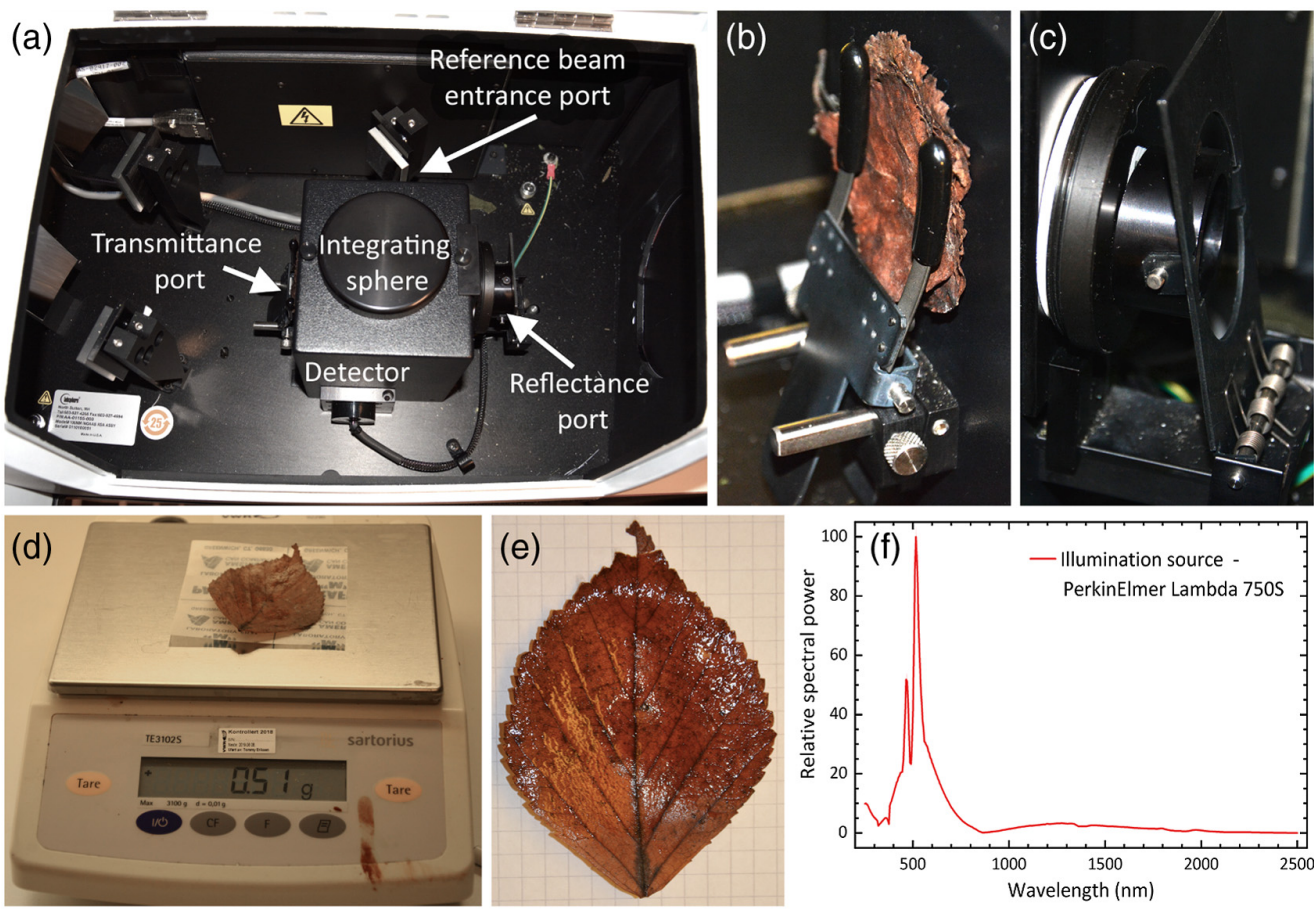

Fig. 1 Experimental setup: (a) spectrophotometer (Lambda 750S, PerkinElmer) with sample set up for transmittance measurements. (b) The samples (moist birch leaves in the image) were fastened by a sample holder, here set up for transmittance measurements. (c) For the transmittance measurements, a Spectralon ${ }^{\circledR} 2^{\prime \prime}$ calibrated diffuse reflectance standard (PerkinElmer) was fastened in the reflectance port by a sample holder. For the reflectance measurements, the reflectance standard was replaced with a sample. The reflectance standard was also used to calibrate the equipment before reflectance or transmittance measurements. (d) The weight of each material was measured on a tabletop scale (TE3102S, Sartorius). (e) A moist birch leaf placed on a grid paper for area measurements where the horizontal and vertical lines are $5 \mathrm{~mm}$ apart. (f) Measured relative spectral power for the spectrophotometer illumination source. 
in Fig. 1. The spectrophotometer had an integrating sphere (60-mm diameter InGaAs integrating sphere, spectralon coating, PerkinElmer Part. No. L6020203) that was applied to imitate the diffuse nature of the radiation illuminating the background through the samples.

The birch leaves studied in this paper were collected in the autumn from the ground (time after tree-detachment was more than a week) after a period with rain, so it is fair to assume that they were close to fully saturated with water. The leaves were brown (i.e., non-chlorophyllic) and had some darker spots [see Fig. 1(f)]. We chose to measure the largest leaves (large enough to cover the beam opening in the spectrophotometer that was $\sim 50 \times 60 \mathrm{~mm}$ ) and the leaves with the smallest and least number of dark spots (i.e., most homogeneous). The dark spots were smaller than $1 \mathrm{~mm}^{2}$ and spread around the whole leaf. We believe that the influence of the dark spots on the spectral measurements is negligible due to the small area of the spots compared to the sample size. All leaves used in this study had dark spots of comparable size and numbers, and we did not observe any significant spectral differences when repeating the spectral measurements on different leaves.

Leaves both have diffuse and specular characteristics where the former mainly stems from multiple scattering effects from the leaf interior while the latter arises at the leaf surface. ${ }^{40}$ The reflection of a leaf usually consists of a high specular peak (glint) confined within a narrow solid angle and a uniform diffuse reflection in the other directions. The spectrophotometer used here could not measure the variation in reflectance/transmittance with observation and illumination directions. Instead, the instrument measured the directional hemispherical reflectance of the leaves, i.e., incident light (from a well-defined angle) reflected in all directions and averaged by the integrating sphere. The overall spectral signature of the leaves measured in this paper is thus a combination of diffuse and spectral components.

After the leaves were collected, the water content of the leaves was saturated in a water bath for $1 \mathrm{~min}$. To prevent large reduction in water moisture (drying) of the leaves, the leaves were stored in a sealed plastic folder before and between measurements. Before each measurement, the leaves were individually suspended in water for $10 \mathrm{~s}$ and then mechanically air-dried for $30 \mathrm{~s}$ by hand. The leaves were then stacked together (for measurements of more than one layer) and fastened in the spectrophotometer. The absolute water content of the leaves used in the study was not measured, however, the method described above ensured that the water content of the leaves were approximately saturated and close to identical before measurements. By measuring the fresh leaf mass $f w$, the oven-dry leaf mass $d w$, and the leaf area $A$, we estimated leaf water content (LWC) and equivalent water thickness (EWT) of the leaves to be: $\mathrm{LWC}=(f w-d w) / f w=79 \% \pm 9 \%$ and $\mathrm{EWT}=(f w-d w) / A=16.5 \pm 1.9 \mathrm{mg} / \mathrm{cm}^{2}$. Before the dry leaf measurements, we dried the leaves in an oven at $105^{\circ} \mathrm{C}$ for $24 \mathrm{~h}$, as recommended in Ref. 41 . The mass and area measurements are described in Sec. 2.2.

In addition to the leaves, the spectral characteristics of three types of textiles were studied: (1) a brown-colored and perforated textile (hereafter referred to as perforated textile \#1 or P1 textile), (2) a green perforated textile (hereafter referred to as perforated textile \#2 or P2 textile), and (3) a white textile without perforations. The P1 textile had larger holes than the P2 textile but fewer holes per area. The mass per area and density of the leaves and textiles were measured (their values are presented in Table 1 together with error estimates).

We cut the samples into square patches of size $\sim 5 \times 5 \mathrm{~cm}$ to fit inside the reflectance or transmittance port (depending on the type of measurement) of the spectrophotometer and cover the beam opening. For the reflectance measurements, a sample holder fastened the samples vertically at the reflectance port of the spectrophotometer (Fig. 1). Most of the reflectance measurements were performed using a black background behind the samples (10 layers of a perforated black textile constituting an opaque background layer). A white background (two layers of a white textile) was used for extra reflectance measurements of single-layered samples. The measurements performed on a white background were carried out to be able to calculate the two-way transmittance using Lillesaeter's model, ${ }^{30}$ as discussed in Sec. 3.2.

The spectrophotometer also allowed for transmittance measurements. During the transmittance measurements, the reflectance port on the integrating sphere (opening used for reflectance measurements) was covered by a white reference standard (PELA9058: calibrated white plate, Spectralon ${ }^{\circledR} .2^{\prime \prime}$ calibrated diffuse reflectance standard, PerkinElmer), while the sample was fastened at the transmittance port [see Fig. 1(a)]. We measured the reflectance and transmittance of 
Table 1 Mass per area $(m / A)$, density $(\rho)$, thickness $(t)$, and estimated errors $(E)$ of the birch leaf and textile samples. The mass per area values have multiple error sources such as weight accuracy, area precision (partial pixel areas and sample curvature), and parallax. The error in sample thickness was estimated by calculating the standard deviation of at least 10 different measurements and added a compressibility error of $\pm 5 \mu \mathrm{m}$ and precision error of $\pm 5 \mu \mathrm{m}$, while the density error of the samples is estimated from the combined errors in mass per area and thickness.

\begin{tabular}{lcccccc}
\hline \hline Sample & $m / A\left(\mathrm{mg} / \mathrm{cm}^{2}\right)$ & $E_{m / a}\left(\mathrm{mg} / \mathrm{cm}^{2}\right)$ & $\rho\left(\mathrm{g} / \mathrm{cm}^{3}\right)$ & $E_{\rho}\left(\mathrm{g} / \mathrm{cm}^{3}\right)$ & $t(\mathrm{~mm})$ & $E_{t}(\mathrm{~mm})$ \\
\hline Moist birch leaf & 20.9 & \pm 1.1 & 1.38 & \pm 0.30 & 0.15 & \pm 0.03 \\
Perf. textile 1 & 28.9 & \pm 1.1 & 0.47 & \pm 0.03 & 0.62 & \pm 0.02 \\
Perf. textile 2 & 11.2 & \pm 0.9 & 0.27 & \pm 0.04 & 0.42 & \pm 0.03 \\
White textile & 9.0 & \pm 0.6 & 0.76 & \pm 0.10 & 0.12 & \pm 0.01 \\
\hline \hline
\end{tabular}

1 to 8 layers for each textile and the birch leaves. With few exceptions, the reflectance and transmittance measurements of each sample were repeated 2 to 3 times. The spectral results were then averaged before they were analyzed in Origin (v. 2019b). Before each measurement series, the spectrophotometer was calibrated by using a white reference background (PELA9058: calibrated white plate, Spectralon ${ }^{\circledR} .2^{\prime \prime}$ calibrated diffuse reflectance standard, PerkinElmer).

\subsection{Mass per Area and Density Estimation}

The mass per area was estimated for each sample. First, we measured the weight of each sample on a tabletop scale (TE3102S, Sartorious, readability $0.01 \mathrm{~g}$, reproducibility $\pm 0.01 \mathrm{~g}$, and linearity $\pm 0.02 \mathrm{~g}$ ) in the laboratory (Fig. 1). We then photographed the samples on $5 \mathrm{~mm}$ grid paper [Fig. 1(e)]. The images were analyzed in Photoshop CS6 (v. 13.1.2 ×64). Through pixel counting, the area of the samples was estimated. Partial pixel areas ( \pm 3 pixels from the sample selection edge) were estimated to be $\sim 0.7 \%$ of the total sample area $\left(\sim 0.6\right.$ to $1.2 \times 10^{7}$ pixels). Measurement errors due to curvature of the samples and parallax (camera lens and grid paper lines not parallel) were estimated to be $<2 \%$ of the total sample area and $<0.7 \%$ of the measured $1 \mathrm{~cm}^{2}$ paper grid area $\left(\sim 2\right.$ to $3 \times 10^{5}$ pixels). The mass per area of each sample was then calculated from the weight and area measurements (presented in Table 1). The presented result for each sample is an average of at least three different weights and area measurements.

The thicknesses of the samples were measured manually with a caliper (TESA Renens Lausanne, 0 to $25 \mathrm{~mm}$ ) with an estimated precision of $\sim 5 \mu \mathrm{m}$. Because the samples were compressible (estimated to $\pm 5 \mu \mathrm{m}$ ), the caliper was tightened to apply a fixed pressure for all samples. To estimate the layer thickness of the samples, we performed at least 10 thickness measurements on a single layer of each sample type (leaves, P1, P2, and white textiles). The measurements were taken at different sample positions, and on 2 to 3 different samples (e.g., different leaves). From the measurements, we then calculated the average thickness value and standard deviation of the samples. The measured thickness and estimated density values (and errors) of the samples are presented in Table 1.

\section{Theoretical Models}

The experimental results presented in this paper have been fitted or compared with three different models. The models are simple to calculate and do not require advanced programming. For the benefit of the reader, we give a brief presentation of each model. For more details, we refer to the literature. ${ }^{25,30}$

\subsection{Extinction Model}

The extinction model was derived by Wilhelm ${ }^{25}$ and used to describe quantitatively the reflectance, transmittance, and absorptance by textiles in terms of specific reflection and absorption 
characteristics of fibers. The model builds on the Stokes equations for transmittance $t_{n}$ and reflectance $r_{n}$ of $n$ plates:

$$
\begin{aligned}
& t_{n}=\frac{c-c^{-1}}{c d^{n}-c^{-1} d^{-n}}, \\
& r_{n}=\frac{d-d^{-n}}{c d^{n}-c^{-1} d^{-n}},
\end{aligned}
$$

where $c$ and $d$ are the constants that only depend on the transmittance and reflectance of the single plates. The equations above were fitted to textile materials of different thicknesses expressed as weight per area by defining $c=1 / \alpha$ and $d=e^{k}$. Here the constant $\alpha$ is the reflectivity of the material when the thickness is taken to the extreme limit, $k$ is the extinction coefficient, whereas the constant $e^{k}$ is the energy lost by absorption in a unit weight per area of material. The extinction coefficient $k$ describes the absorptance characteristics of thin materials and can be considered a spectral fingerprint of the material. Substituting $n$ with $w$, which is the weight per unit area, and using the new definitions yield: ${ }^{25}$

$$
\begin{gathered}
t_{w}=\frac{\left(1-\alpha^{2}\right) e^{-k w}}{1-\alpha^{2} e^{-2 k w}}, \\
r_{w}=\frac{\alpha\left(1-e^{-2 k w}\right)}{1-\alpha^{2} e^{-2 k w}} .
\end{gathered}
$$

The absorptance can then be calculated from the following relationship: $a_{w}=1-r_{w}-t_{w}$.

It is often necessary to measure samples with an underlying background. The spectral characteristics of the background must then be included, especially if the sample has high transparency. If the reflectance of the background is given by $\beta$, expressions for the reflected, transmitted, and absorbed light of the system (sample and background) is given by ${ }^{25}$

$$
\begin{gathered}
\left(\frac{I_{r}}{I_{0}}\right)_{\text {syst. }}=r_{w}+t_{w}^{2} \beta+t_{w}^{2} r_{w} \beta^{2}+t_{w}^{2} r_{w}^{2} \beta^{3}+\ldots=r_{w}+\frac{t_{w}^{2} \beta}{1-r_{w} \beta} \\
\left(\frac{I_{t}}{I_{0}}\right)_{\text {syst. }}=t_{w}(1-\beta)+t_{w} r_{w} \beta(1-\beta)+t_{w} r_{w}^{2} \beta^{2}(1-\beta)+t_{w} r_{w}^{3} \beta^{3}(1-\beta) \ldots=\frac{t_{w}(1-\beta)}{1-r_{w} \beta}, \\
\left(\frac{I_{a}}{I_{0}}\right)_{\text {syst. }}=1-\left(\frac{I_{r}}{I_{0}}\right)_{\text {syst. }}-\left(\frac{I_{t}}{I_{0}}\right)_{\text {syst. }}=\frac{a_{w}(1+\beta)\left(t_{w}-r_{w}\right)}{1-r_{w} \beta}
\end{gathered}
$$

\subsection{Lillesaeter Model}

A simpler model (compared to the extinction model) on the reflectance of multi-layered samples was presented by Lillesaeter. ${ }^{30}$ Lillesaeter considered the reflectance of a single-layered sample placed on a background, which can be expressed as

$$
r_{1}=r+\beta \tau^{2},
$$

where $r$ is the sample reflectance and $\beta$ is the reflectance of the background. By measuring the reflectance of a single-layered sample on a dark and light background (background reflectance $\beta_{D}$ and $\beta_{L}$, respectively), one get the following relationships:

$$
\begin{aligned}
& r_{1 L}=r+\beta_{L} \tau^{2}, \\
& r_{1 D}=r+\beta_{D} \tau^{2},
\end{aligned}
$$

which can be used to find the reflectance of the sample $r$ and the two-way transmission: ${ }^{30}$ 


$$
\tau^{2}=\frac{r_{1 L}-r_{1 D}}{\beta_{L}-\beta_{D}}
$$

The two-way transmission and the reflectance of the sample can then be used to estimate the reflectance of $n$-layered samples with the following equation:

$$
r_{n}=r\left[1+\tau^{2}+\ldots \tau^{2(N-1)}\right]+\beta \tau^{2 N}
$$

\subsection{Multiple Scattering Model}

The Lillesaeter model (presented in Sec. 3.2) does not account for multiple scattering between the sample and the background [Eq. (8)]. Multiple scattering is expected to have a considerable contribution to the measured spectral characteristics of thin material samples that are transparent, especially when the background has a high reflectance. Multiple scattering should therefore be implemented in a realistic model.

When multiple scattering between the sample (reflectance $r$ ) and background (reflectance $\beta$ ) is included, the reflectance of the system (sample and background) is expressed by

$$
r_{m 1}=r+\beta \tau^{2}+r \beta^{2} \tau^{2}+r^{2} \beta^{3} \tau^{2}+\ldots=r+\frac{\beta \tau^{2}}{1-r \beta} .
$$

The total reflectance measured from a single-layered sample placed on the light and dark background is then

$$
\begin{aligned}
& r_{L}=r+\frac{\beta_{L} \tau^{2}}{1-r \beta_{L}}, \\
& r_{D}=r+\frac{\beta_{D} \tau^{2}}{1-r \beta_{L}} .
\end{aligned}
$$

These equations [Eqs. (14) and (15)] can then be combined and solved for the sample reflectance and the two-way transmission:

$$
\begin{gathered}
r=\left[1+r_{L} \beta_{L}-\sqrt{1+4 \tau^{2} \beta_{L}^{2}-2 r_{L} \beta_{L}+r_{L}^{2} \beta_{L}^{2}}\right] / 2 \beta_{L}, \\
\tau^{2}=\left[\left(\beta_{D}-\beta_{L}\right)\left(\beta_{L} r_{D}-1\right)\left(r_{D}-r_{L}\right)\left(\beta_{D} r_{L}-1\right)\right] /\left[\beta_{L}+\beta_{D}\left(-1+\beta_{L}\left(r_{D}-r_{L}\right)\right)\right]^{2} .
\end{gathered}
$$

The mathematical expressions given in Eqs. (16) and (17) constitute modified expressions, of the original Lillesaeter model, as given in Eqs. (11) and (12), thereby augmenting the model to capture more of the underlying reflectance mechanisms of thin material samples.

\section{Spectral Properties of Multiple Layers of Leaves and Textiles}

In this section, we present experimental results from the spectral measurements on 1 to 8 layers of moist birch leaves and textiles between 250 and $2500 \mathrm{~nm}$. The absorptance $a$ of each sample was calculated using the relationship: $a=1-r-t$, where $r$ and $t$ are the measured reflectance and transmittance values, respectively.

\subsection{Moist Birch Leaves}

Figure 2 shows the spectral reflectance, transmittance, and absorptance of multiple layers (1 to 8) of dead birch leaves that were moistened by water to the point of saturation. The reflectance and transmission values of the moist birch leaves (Fig. 2) were highest in the following wavelength bands: 800 to $1400 \mathrm{~nm}, 1600$ to $1850 \mathrm{~nm}$ and had a smaller top in the 2100 to $2400 \mathrm{~nm}$ band. In 


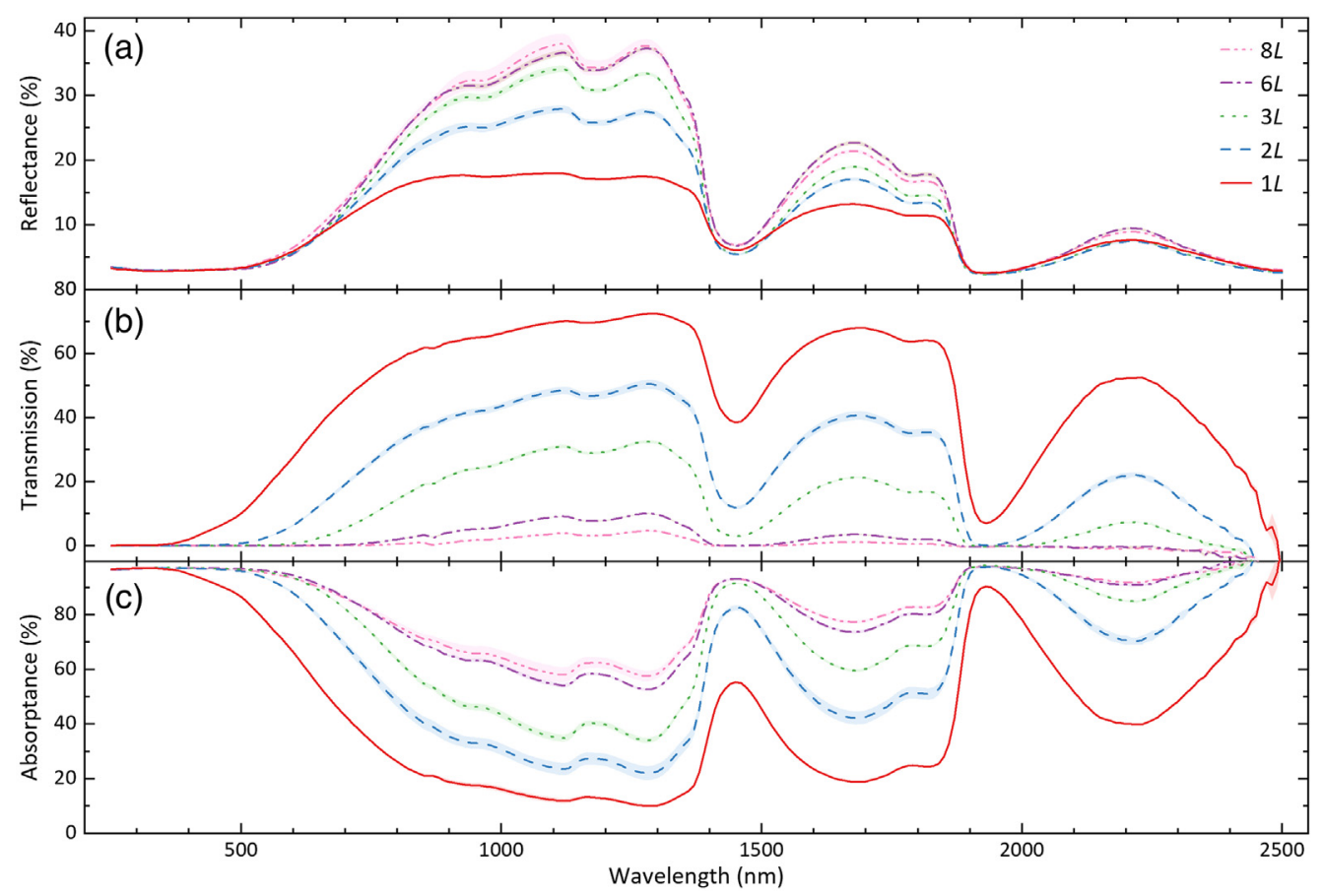

Fig. 2 Spectral properties of one (red solid), two (blue dashed), three (green dotted), six (violet dash-dotted), and eight (pink dash-dot-dotted) layers of moist birch leaves: experimental data (mean values) on (a) reflectance, (b) transmission, and (c) absorptance values plotted against wavelength. The semitransparent areas around the curves represent SEM values of the data (errors smaller than the lines might not be visible in the plot).

the visible region, leaf pigments predominantly absorb most of the incoming light, ${ }^{42}$ while from $1300 \mathrm{~nm}$ (SWIR), the reflectance and transmission are attenuated by the water content of the leaf. ${ }^{40}$ We found that the reflectance of the wet leaves was markedly reduced around 1450 and $1950 \mathrm{~nm}$. Liquid water has absorption peaks of increasing size around 970, 1200 1450, and $1950 \mathrm{~nm},{ }^{43}$ which corresponds well with the absorptance plot of the birch leaves [Fig. 2(c)].

The leaves studied in this work were brown and detached from the tree for a significant time ( 1 to 2 weeks). When a leaf senesces and decays, e.g., after being removed from a tree, the plant tissues degrade, pigments break down, and the enzymatic activity in the leaf increases. ${ }^{44}$ This dramatically changes the optical properties of the leaf and is easily assessable visually as the leaf change to autumn colors. The birch leaves studied in this work for instance (Betula pendula), changed color from green to yellow to brown during the senescence process. Green autumn birch leaves typically contain 40 to $50 \mu \mathrm{g}$ chlorophyll $\mathrm{per}_{\mathrm{cm}}^{2}$, whereas yellow leaves before falling have a chlorophyll concentration $<10 \mu \mathrm{g} / \mathrm{cm}^{2} .{ }^{45}$ Due to reduced chlorophyll concentration, yellow birch (Betula pendula) leaves reflect all colors of visible light more strongly than green leaves; up to twice as much light compared to that of the green leaves at $550 \mathrm{~nm}$. ${ }^{46}$ We did not observe the typical reflectance peak for green leaves around $550 \mathrm{~nm}$, nor a steep reflectance increase from VIS to NIR wavelengths, usually in the wavelength range $\sim 670$ to $770 \mathrm{~nm}$ [Fig. 2(a)]. This indicates that the chlorophyll concentration in the measured leaves was low.

Between 750 and $1450 \mathrm{~nm}$, the reflectance in a leaf is affected by alterations in the leaf intercellular structure, whereas components such as water, cellulose, protein, nitrogen, and lignin mostly affect the reflectance in SWIR regions. ${ }^{47}$ Hovi et al. ${ }^{48}$ studied the seasonal trends of silver birch leaves (Betula pendula) and found that the reflectance of silver birch leaves increased markedly during autumn in the visible range, while slowly but steadily during the summer and autumn in NIR and SWIR. We could not find any reported studies on the spectral properties of silver birch leaves (as used in this work) following tree detachment, however, there are some studies on the reflectance of paper birch leaves $72 \mathrm{~h}$ following branch cutting. ${ }^{49}$ In the $72-\mathrm{h}$ period, few changes in reflectance were observed in VIS, however, the reflectance in NIR increased markedly due to dehydration. Foley et al. ${ }^{50}$ investigated the leaf reflectance of five 
tropical trees that were clipped and thereafter assessed regularly for up to 7 days. In the visible spectrum, leaf reflectance did not change until wilting of the leaf was observed, whereas the NIR and SWIR wavelength regions were affected immediately by small changes in water content (caused by structural changes within the leaf and by changes in light absorption by water). Although the birch leaves measured in our work were detached from the tree for a significant time, the water content of the leaves (estimated to $79 \% \pm 9 \%$ ) was still high due to rainy days and low temperatures $\left(0^{\circ} \mathrm{C}<10^{\circ} \mathrm{C}\right)$ while the leaves were on the ground, and due to water saturation of the leaves in the lab before measurements. The high water content dominates the SWIR regions of the spectra with deep reflectance (and transmittance) valleys and high absorptance values (Fig. 2). We speculate that the effect of water on the measured reflectance in SWIR is higher than other degrading effects (intercellular structure and content), however, this should be investigated in future works.

As the effective leaf thickness (and thereby any potential and real-world canopy depths) increased, light in the visible spectrum (400 to $700 \mathrm{~nm}$ ), between 1400 and $1500 \mathrm{~nm}$ and 1900 and $2500 \mathrm{~nm}$ did not transmit significantly beyond the first two leaves. Correspondingly, we see that the number of stacked leaves did not affect the measured reflectance of the leaves much in the same wavelength bands. For other wavelengths bands, (700 to $1400 \mathrm{~nm}$ and 1500 to $1900 \mathrm{~nm}$ ), on the other hand, light was transmitted through the leaf stack until the number of leaves reached seven or eight. The transmission through a stack of eight leaves [pink dash-dotdotted line, Fig. 2(b)] is $<5 \%$, i.e., the two-way transmission of the sample is $<0.25 \%$. Lillesaeter ${ }^{30}$ and Berard et al. ${ }^{29}$ also found that 7 to 8 layers of green birch leaves were necessary to reduce most of the transmission through the leaf stack.

In the same wavelength range, (700 to $1400 \mathrm{~nm}$ and 1500 to $1900 \mathrm{~nm}$ ), the wet birch leaves also reflected more light as the number of stacked leaves increased. For instance, the measured reflectance of a single-layer birch leaf [red solid line, Fig. 2(a)] was around $18 \%$ at $1000 \mathrm{~nm}$, while for a stack of 8 birch leaves [pink dash-dot-dotted line, Fig. 2(a)] at the same wavelength, the measured reflectance was found to be around $32 \%$. That is, the observed increase in reflectance most likely occurred because the black (and low-reflective) background contributed less to the experimental reflectance data being captured as the number of leaves in the stack increased.

Our findings confirm the hypothesis that objects located underneath canopies with water saturated leaves and with a leaf area index (LAI) of $<2$ (i.e., an effective thickness less than two leaf layers) may potentially be revealed because a significant amount of light is transmitted through leaves in NIR and SWIR and back to any external remote sensor. In a deciduous canopy (with brown leaves) that is effectively more than three leaf layers thick (assuming a homogeneous LAI > 3), most of the light in the visible spectrum will be absorbed or reflected by the leaf material, and only small portions of the visible light will be transmitted. LAI values between 3 and 4 are typical for horizontal-leafed species, and generally, light transmission in canopies decreases fast with LAI to around $10 \%$ once LAI is larger than $4 .{ }^{51}$ Although a canopy has a high LAI value, LAI varies across space due to variable tree spacing and leaf arrangements. As a consequence, there might be canopy areas with gaps between leaves where a larger portion of the light transmits and reach the ground beneath. Conversely, there might also be canopy areas where more leaves overlap resulting in a higher LAI. Canopies also have larger distances between the constituting leaves than what was measured in this study (leaves in contact with each other). We speculate that an increased distance between the leaves will yield more light scattering and decreased transmission through the leaves. The effect should be investigated in future studies.

For camouflage purposes, we note that the spectral reflectance in the visual range of an object (and hereby the color appearance to any observer) will not be much affected under a canopy where the object is covered by at least two leaf layers (i.e., a homogeneous LAI $>2$ ). The first single leaf layer in a canopy, when viewed from above/outside, will constitute effective concealment to any object hiding underneath it because the reflectance of such a leaf canopy, when viewed from any outside observer, will keep its color appearance regardless of the number of leaves in the canopy exceeding two. The visible light reaching a canopy of birch leaves, as measured in this experiment, will be attenuated by the first and second leaf layers. Any visible reflectance signatures that are captured (e.g., by a sensor) will therefore be more dominated, and more indicative, by the top layers of a canopy (or any leaf coverages) of a hidden object. 
In the NIR and SWIR range, however, there is a greater risk (to any object hiding underneath vegetation or canopies) that incident light reaching, e.g., a canopy, will be able to penetrate the leaves, interact with any object underneath, and be reflected back through the canopy to any external sensor. This means that information received by a sensor in those wavelength bands (700 to $1400 \mathrm{~nm}$ and 1500 to $1900 \mathrm{~nm}$ ) is more likely, than in the visible band, to be a mix of canopy signatures and object/background signatures. There will thus be large differences in canopy penetration depth between the visible wavelengths and the NIR wavelengths, as we have also seen in the experimental results in this study.

Such differences in canopy penetration depths are due to differences in (i) wavelengths and (ii) effective numbers of leaf layers. The differences are also expected to be of relevance for remote sensing purposes where vegetated areas are continuously mapped and classified, and where differences in red light reflectance and corresponding NIR light are used as spectral vegetation indices. $^{29,52-54}$

\subsection{White Textile}

Figure 3 presents the spectral characteristics of the white textile. Compared to a moist birch leaf, the textile is more reflective (reflectance up to 94\%), and less transparent over most of the measured wavelengths. Except for the UV (under $380 \mathrm{~nm}$ ) and longest wavelengths (over $2100 \mathrm{~nm}$ ), the absorptance of the textile was low $(<10 \%)$. The reflectance and transmission of the textile are thus varying little over these wavelengths. In the SWIR range, the biggest change in the textile's reflectance and transmission occurs around $1660 \mathrm{~nm}$ where there is a characteristic valley, i.e., an absorption top.

Similar to the birch leaves, 7 to 8 layers of the textile is sufficient to hinder reflectance contributions from the background at NIR and SWIR wavelengths: the reflectance values measured from a stack of 6 layers of the textile [violet dash-dotted line, Fig. 3(a)] is similar to that of a stack with 8 layers [pink dash-dot-dotted line, Fig. 3(a)]. The textile is not perforated, however, it was

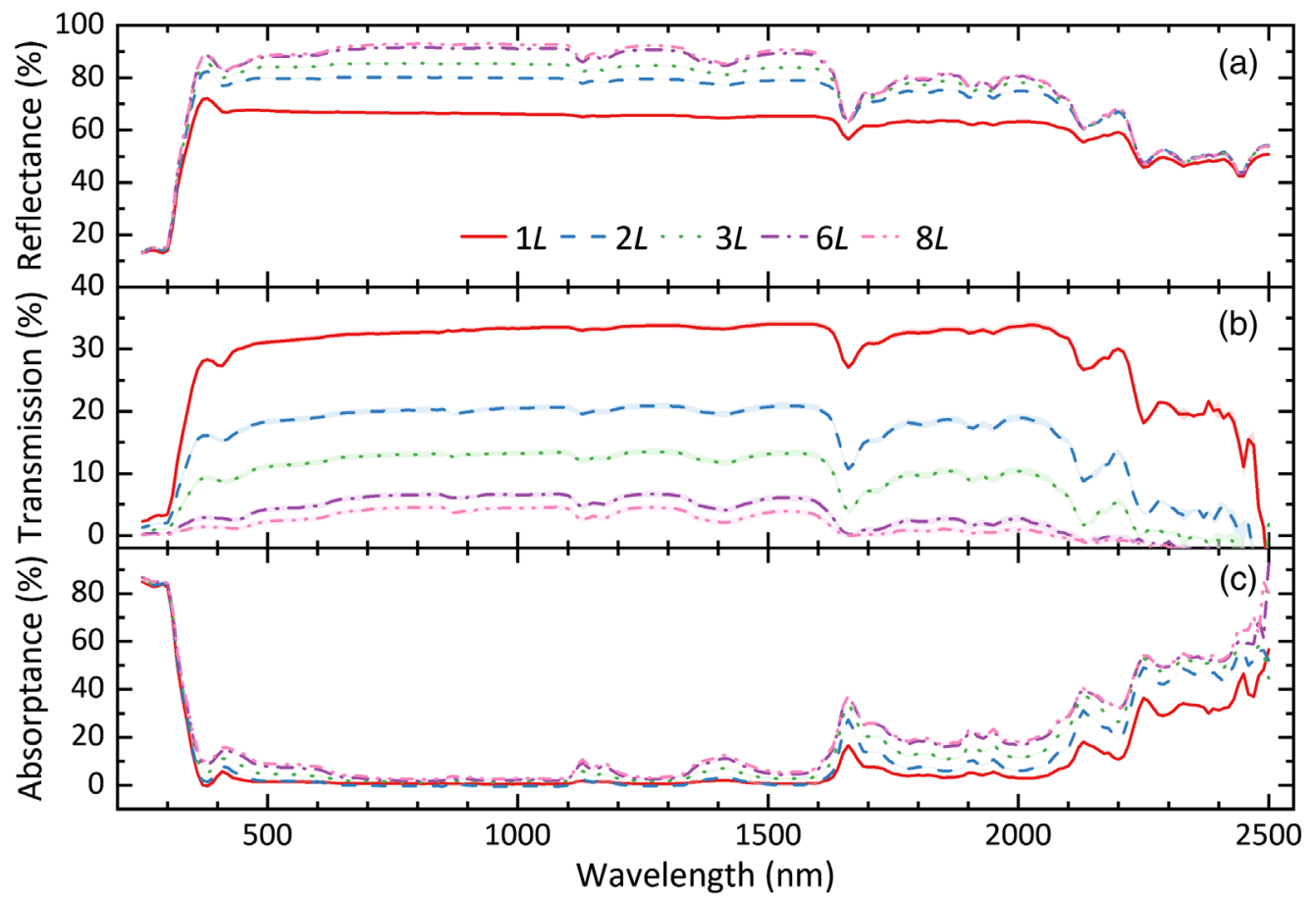

Fig. 3 Spectral properties of one (red solid), two (blue dashed), three (green dotted), six (violet dash-dotted), and eight (pink dash-dot-dotted) layers of the white textile sample: Experimental data (mean values) on (a) reflectance, (b) transmission, and (c) absorptance plotted against wavelength. The semitransparent areas around the curves represent SEM values of the data (errors smaller than the lines might not be visible in the plot). 
Mikkelsen and Selj: Spectral characteristics of moist birch leaves and synthetic materials...

the thinnest of the three textiles with a thickness of about $0.12 \mathrm{~mm}$. In comparison, the moist birch leaves had a thickness of about $0.15 \mathrm{~mm}$ (Table 1). Because of the thin layers, a high number of white textile layers were necessary to significantly reduce the transmission of light.

The absorptance data for the textile [Fig. 3(c)] peaked around the following wavelengths: $300,420,1150,1400,1660,2150,2250$, and $2450 \mathrm{~nm}$. Although the absolute absorptance of the textile is low (compared to the reflectance and transmission) in the 380- to 2100-nm range, the relative change in absorptance is significant when the number of layers is increased. As an example, at $1000 \mathrm{~nm}$, the absorptance of a single layer of the white textile is around $0.5 \%$, while for 8 layers, the absorptance is more than $4 \%$.

\subsection{Perforated Textile \#1}

We found that the reflectance of a single layer of the perforated textile \#1 (abbreviated P1) varied little (reflectance value around 40\%) between 800 and $2200 \mathrm{~nm}$ [Fig. 4(a)]. In the UV and VIS wavelength bands, the reflectance of the textile decreased toward zero with shorter wavelengths. This is similar to the reflectance characteristics measured for the birch leaf [Fig. 2(a)] that reflected little light at these wavelengths due to pigment absorption.

We found that 2 to 3 layers of the P1 textile were sufficient to hinder significant reflection from the background and back through the textile. The transmission of a single layer of the P1 textile was around $27 \%$ at $1000 \mathrm{~nm}$, whereas for two layers of the textile, the transmission was reduced to $6 \%$. One reason for the large transmission decrease might be due to the textile's high mass per area (Table 1). Although the textile is perforated, the textile is thick (around $0.62 \mathrm{~mm}$ ) compared to the other textiles and the moist birch leaves. The thickness of the textile and the high material absorption [Fig. 4(c)] ensured that little light was transmitted through a stack of two layers of the P1 textile. Because the effective area of the small holes was (much) less than the area of the remaining solid textile material, it is likely the number of free light pathways through the material was effectively reduced when the number of layers exceeded one. Compared to a

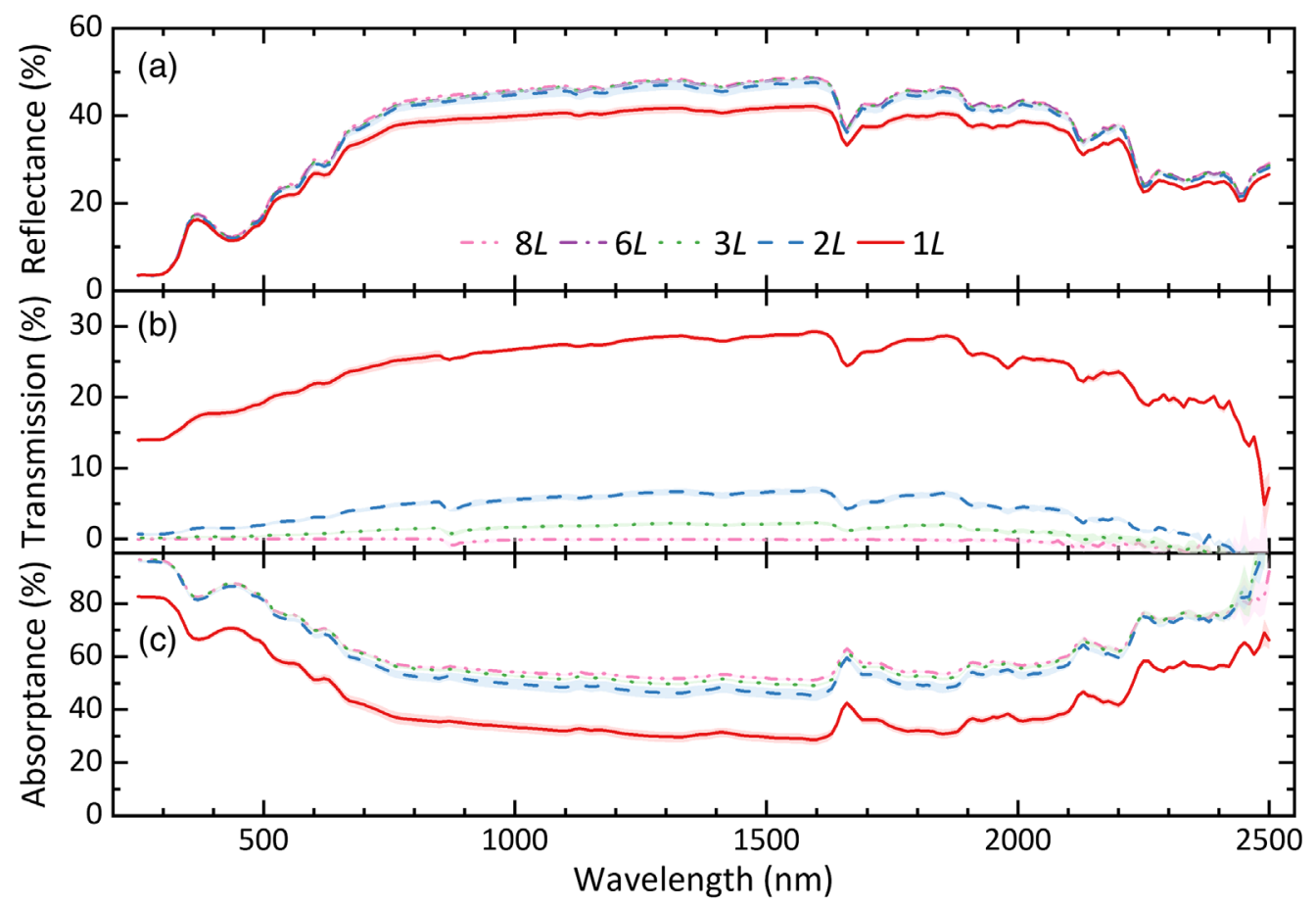

Fig. 4 Spectral properties of one (red solid), two (blue dashed), three (green dotted), six (violet dash-dotted), and eight (pink dash-dot-dotted) layers of the perforated textile \#1 sample: Experimental data (mean values) on (a) reflectance, (b) transmission, and (c) absorptance plotted against wavelength. The semitransparent areas around the curves represent SEM values of the data (errors smaller than the lines might not be visible in the plot). 
single layer of the white textile [red solid line, Fig. 3(b)], a single layer of the P1 textile transmitted slightly less light [red solid line, Fig. 4(b)] for all measured wavelengths larger than $350 \mathrm{~nm}$. Except for the large absorptance bands of water (around 1450 and $1950 \mathrm{~nm}$ ) and leaf pigments (UV-VIS wavelengths), a single layer of the white or the P1 textile transmitted less light than brown and moistened birch leaves.

\subsection{Perforated Textile \#2}

Figure 5 presents the spectral properties of the perforated textile \#2 (referred to as P2). Except the prominent reflectance valleys observed for a single-moist birch leaf (caused by water absorbance), the reflectance spectra of a single layer of the P2 textile [red solid line, Fig. 5(a)] resembles that of the moist birch leaf [red solid line, Fig. 2(a)]. Similar to a single layer of birch leaf, the measured reflectance of a layer of the $\mathrm{P} 2$ textile is low $(<10 \%)$ and increases with the wavelength in the UV and VIS bands. At longer wavelengths, the reflectance of the P2 textile varied little around $20 \%$.

Unlike the birch leaves, the P2 textile required only 3 to 4 layers (compared to 7 to 8 layers for the leaves) to hinder most of the reflectance from the background at NIR and SWIR wavelengths. For example, at $1000 \mathrm{~nm}$, the transmission through 3 layers of the P2 textile was 10\% [green dotted line, Fig. 5(b)], yielding a two-way transmission of only $1 \%$. At NIR and SWIR wavelengths, a single layer of the P2 textile [red solid line, Fig. 5(b)] transmitted considerably less light than a single-layer birch leaf [red solid line, Fig. 2(b)].

Both the P1 and P2 textiles studied in this paper were perforated with small holes and were relatively thick: 0.62 and $0.42 \mathrm{~mm}$, respectively. Moreover, the mass per area of the P1 textile is more than twice that of the $\mathrm{P} 2$ textile (Table 1). We believe that the difference in sample thickness is one of the main reasons why the P1 textile transmitted less light compared to the P2 textile [Figs. 4 and 5(b)]. We discuss the spectral characteristics of the textiles and birch leaf in Sec. 5 where their corresponding reflectance, transmission, and absorptance data are plotted together

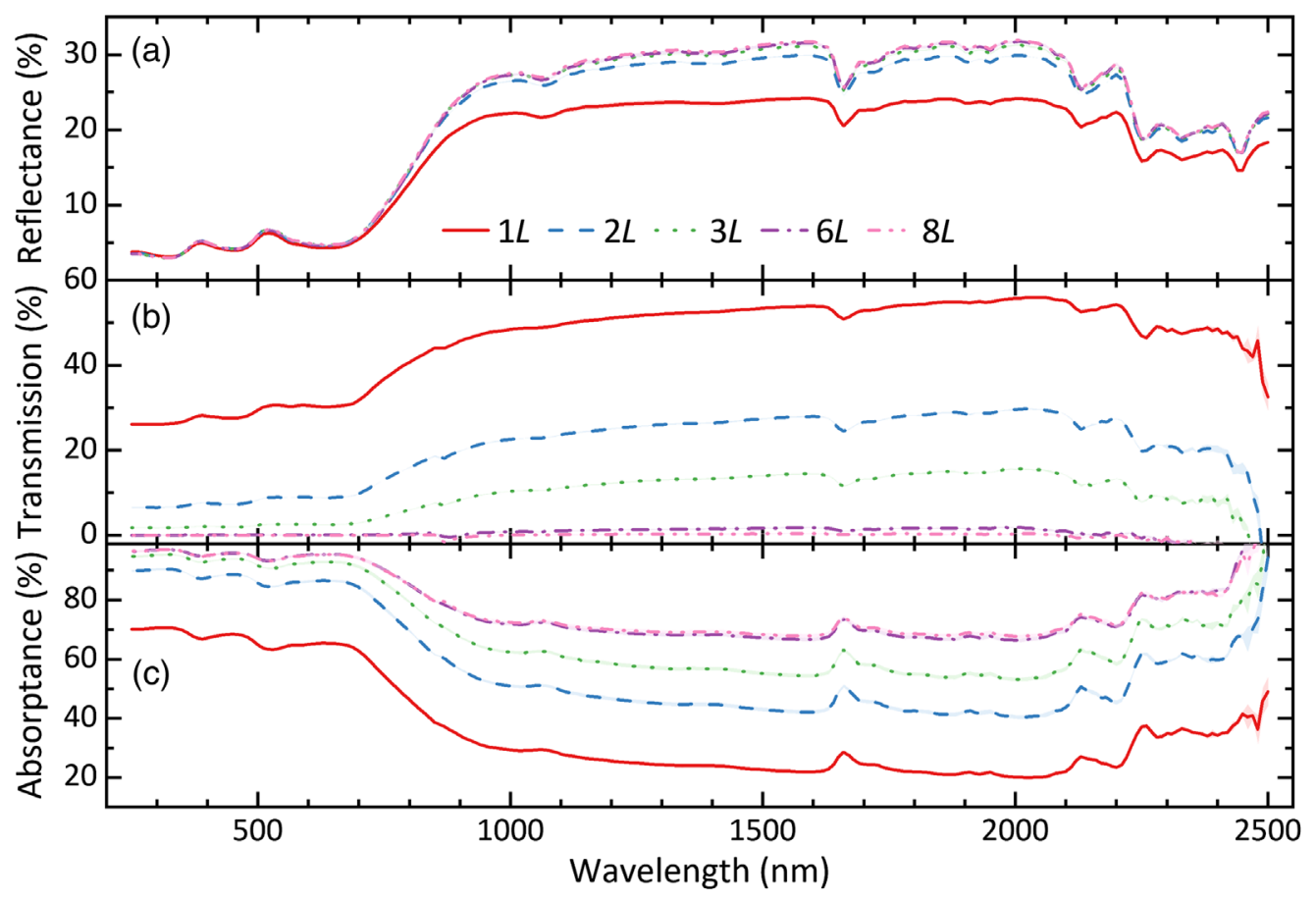

Fig. 5 Spectral properties of one (red solid), two (blue dashed), three (green dotted), six (violet dash-dotted), and eight (pink dash-dot-dotted) layers of the perforated textile \#2 sample: Experimental data (mean values) on (a) reflectance, (b) transmission, and (c) absorptance plotted against wavelength. The semitransparent areas around the curves represent SEM values of the data (errors smaller than the lines might not be visible in the plot). 
versus mass per area, yielding the possibility to compare experimental data and the mathematical extinction model.

\section{Spectral Properties versus Mass Per Area}

At some selected wavelengths $(700,1000$, and $1450 \mathrm{~nm})$, chosen by the authors as they show different physics, we show the reflectance, transmittance, and absorptance data of the moist birch leaf and the textiles as a function of their corresponding mass per area, enabling us to compare different types of sample materials directly. We chose the wavelengths to represent different sensor bands, i.e., $700 \mathrm{~nm}$ for VIS light detected by the eye, $1000 \mathrm{~nm}$ for NIR light sensed by night vision sensors, and $1450 \mathrm{~nm}$ for SWIR light and corresponding with one of the water absorption peaks. The data have been fitted to the extinction model (presented in Sec. 3.2), i.e., Eqs. (5)-(7). The fitting parameters of the model are the sample reflectivity $\alpha$, describing reflectance characteristics of a sample when the thickness is very large, and the extinction coefficient $k$. A higher extinction coefficient value implies that the absorptance is large per mass per unit area, i.e., the transmission decreases fast with an increase in mass per area (corresponding with an increased number of layers for each specific material type).

\subsection{Visible Light: $700 \mathrm{~nm}$}

For incoming light at $700 \mathrm{~nm}$, we found that the extinction model fitted well with the spectral characteristics of the birch leaf and the textiles (Fig. 6). The biggest difference between the model and experimental data occurred for single-layered samples (small mass per area) where the model usually predicted slightly higher reflectance values than the data and slightly smaller transmission values than the transmission data. At $700 \mathrm{~nm}$ and for samples with similar mass per area, we found a significant difference in the measured reflectance between the various sample types. The measured reflectance ranged from around $6 \%$ for the P2 textile [green circles, Fig. 6(a)] to $93 \%$ for the white textile [violet stars, Fig. 6(a)]. The reflectance of the P2 textile was closest to that of the moist birch leaf, which reflected little light at this wavelength due to leaf pigment absorption. ${ }^{40}$

The reflectance of all the samples except the white textile varied little with mass per area, however, the reflectance of the white textile seemed to reach a plateau around $90 \mathrm{mg} / \mathrm{cm}^{2}$. We would probably observe a more significant change in reflectance of the other by measuring thinner samples. It is also worth mentioning that the difference in reflectance between the samples and background (black) was the largest for the white textile. By choosing a white background,

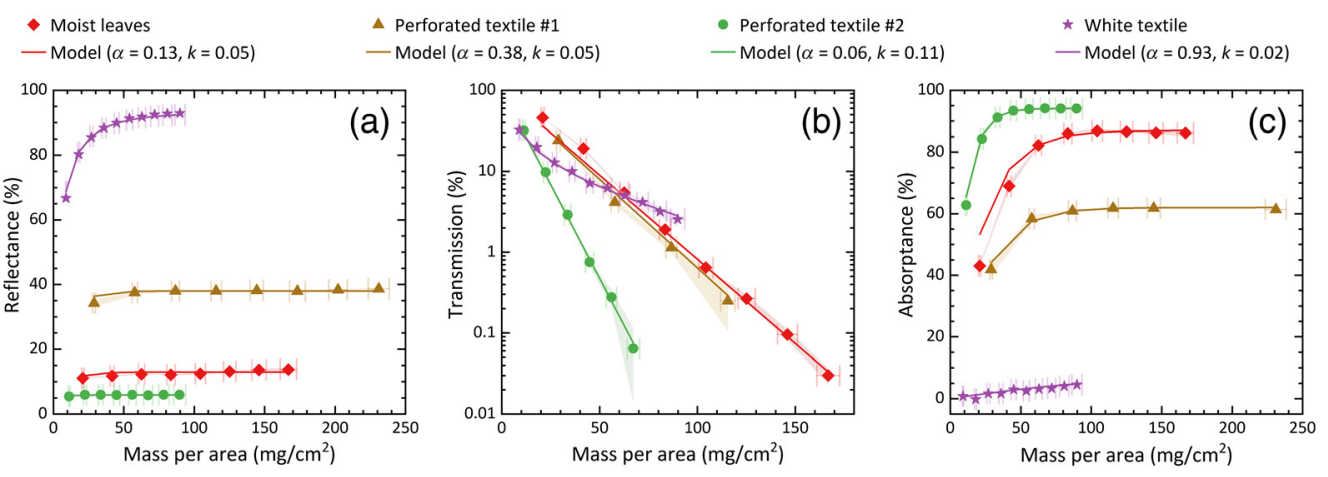

Fig. 6 Spectral properties versus mass per area for moist birch leaves (red squares), perforated textile \#1 (sand triangles), perforated textile \#2 (green circles), and white textile (violet stars) samples at $700 \mathrm{~nm}$ : measured (a) reflectance, (b) transmission, and (c) absorptance of the samples (mean values) plotted against mass per area. The data points are fitted to the extinction model for each sample (solid lines) with different reflectance coefficients $(\alpha)$ and extinction coefficients $(k)$ with dimension $\mathrm{cm}^{2} / \mathrm{mg}$. The semitransparent areas around the curves represent SEM values of the data (errors smaller than the lines might not be visible in the plot), while the horizontal error bars represent the error of the samples' mass per area. 
we would probably observe a smaller increase in reflectance of the white textile versus mass per area and a larger increase in reflectance for the other samples. Regardless of the reflectance of the background, we expect the samples to reach a plateau around the same mass per area when the transmittance of the samples is the same.

Interestingly, the white textile was also the only sample without a constant exponential relationship between the transmission and mass per area [Fig. 6(b)]. We speculate that this is because the white textile was very thin and had a high reflectance, resulting in multiple-reflection effects. For instance, through the white textile, we cannot neglect the intensity of the light that has the following optical patch: transmission through the first layer, reflection at the second layer, then reflection at the back of the first layer, and followed by reflection at the second layer again before transmitting through the surface of the first layer. Based on the reflectivity and transmission of a single layer of the white textile, we estimate the intensity of this double-reflected light at $700 \mathrm{~nm}$ to: $I_{\mathrm{wt} \_\mathrm{m} 2}(700 \mathrm{~nm})=t_{\mathrm{wt} 700}^{2} r_{\mathrm{wt} 700}^{3} \approx 0.33^{2} \times 0.93^{3}=0.09=9 \%$.

At $700 \mathrm{~nm}$, we found that the $\mathrm{P} 2$ textile had the largest absorptance, almost 95\%, whereas the white textile absorbed the least, around 7\% [Fig. 6(c)]. Here the absorptance of the white textile increased linearly with the mass per area, unlike the other samples that reached a plateau around $100 \mathrm{mg} / \mathrm{cm}^{2}$ (corresponding to 3 to 4 layers) for the birch leaf and the P1 textile, and around $40 \mathrm{mg} / \mathrm{cm}^{2}$ (corresponding to 3 to 4 layers) for the P2 textile. We refer to these values as the absorption threshold value $A_{\max }(\lambda)$ which is expected to be wavelength-dependent for most samples. The transmission through these samples at $A_{\max }(700 \mathrm{~nm})$ was $<1 \%$ [Fig. $\left.6(\mathrm{~b})\right]$.

The P2 textile had the largest extinction coefficient $k=0.11$, whereas the white textile had the smallest extinction coefficient $k=0.02$. This means that at $700 \mathrm{~nm}$, the transmission of the P2 textile decreased most when the mass per area was increased. Although the reflectance values of the P1 textile at $700 \mathrm{~nm}$ were much lower than those of the birch leaf, the P1 textile had the same extinction coefficient $k=0.05$ and the same transmission curve as the birch leaf at this wavelength [Fig. 6(b)]. In the VIS region and particularly at the red-end of the spectrum, the P1 textile is thus thought to be a promising material candidate for camouflage applications, as it resembles important transmission characteristics. If necessary, the reflectance of the textile can also be adjusted (e.g., using different dyes) to resemble the reflectance of the desired background, hence reducing revealing spectral reflectance contrasts between object and the background.

Around $60 \mathrm{mg} / \mathrm{cm}^{2}$, corresponding to almost three layers of moist birch leaves (Table 1), the white textile had the same transmission value as the leaf (and the P1 textile). It is potentially useful to have a material with properties such as the white textile where the transmission can be tailored to mimic the transmission of natural biomaterial samples (e.g., leaves) by changing the mass per area, i.e., effectively changing the thickness of a layer (or layers) of a given material.

\subsection{NIR: $1000 \mathrm{~nm}$}

In the NIR region and compared to the measurements at $700 \mathrm{~nm}$ [Fig. 6(a)], we found that all the samples, except the white textile, had a higher change in reflection when the mass per area was increased [Fig. 7(a)]. We believe that this difference was mainly due to the increased transmission through these samples at $1000 \mathrm{~nm}$ compared to the transmission at $700 \mathrm{~nm}$. For incoming light of $1000 \mathrm{~nm}$, the reflectance of the P2 textile is closest to that of the birch leaf, with reflectance values around $20 \%$ to $30 \%$. At $50 \mathrm{mg} / \mathrm{cm}^{2}$ (corresponding to 2 to 3 leaf layers), the reflectance of the birch leaf and the P2 textile overlap.

At $1000 \mathrm{~nm}$, none of the textiles transmitted light similar to birch leaves [red squares, Fig. 7(b)]. The textiles had lower transmission or a higher extinction coefficient than the leaves (meaning that the transmission decreases faster when the mass per area increases). For example, the white textile [violet stars, Fig. 7(b)] had the same extinction coefficient as the birch leaf, but the transmission values are lower and the curve shape is non-linear (as discussed in the previous section). Based on the linear fit by the extinction model, an extrapolation of the data suggests that a thinner sample (smaller mass per area) of the P1 textile [brown triangles, Fig. 7(b)] could transmit light similar to a single layer of the moist birch leaf.

The extinction model fit well with all our data measured at $1000 \mathrm{~nm}$. As mentioned earlier, the most significant differences between the model and the data were observed for single-layered 
Mikkelsen and Selj: Spectral characteristics of moist birch leaves and synthetic materials...
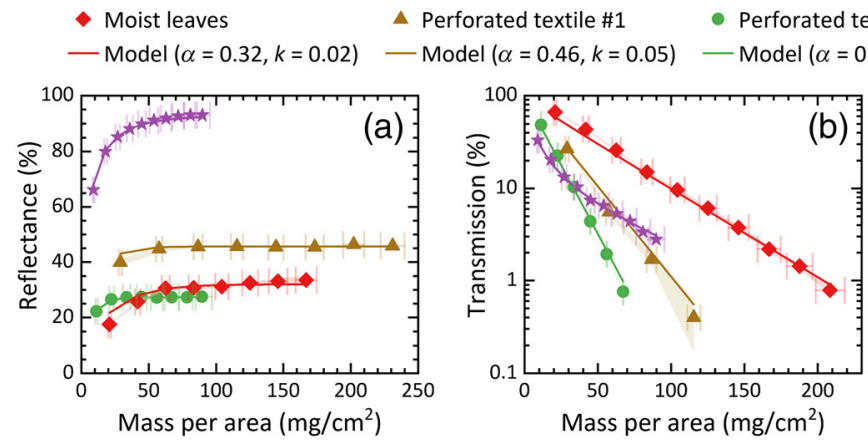

$\star$ White textile

-Model $(\alpha=0.93, k=0.02)$

Fig. 7 Spectral properties versus mass per area for moist birch leaves (red squares), perforated textile \#1 (sand triangles), perforated textile \#2 (green circles), and white textile (violet stars) samples at $1000 \mathrm{~nm}$ : measured (a) reflectance, (b) transmission, and (c) absorptance of the samples (mean values) plotted against mass per area. The data points are fitted to the extinction model for each sample (solid lines) with different reflectance coefficients $(\boldsymbol{\alpha})$ and extinction coefficients $(k)$ with dimension $\mathrm{cm}^{2} / \mathrm{mg}$. The semitransparent areas around the curves represent SEM values of the data (errors smaller than the lines might not be visible in the plot), while the horizontal error bars represent the error of the samples' mass per area.

samples (small mass per area), where the model usually estimated slightly higher reflectance values and lower transmission values than the experimental data.

For all samples except the white textiles, the absorptance at $1000 \mathrm{~nm}$ increased with mass per area until a plateau was reached (as the transmission decreased towards zero). We did not observe an absorptance plateau for the white textile. That is probably because we did not measure sufficient number of the textile layers to reduce the transmittance through the textile stack to values below $1 \%$, this value seems to be characteristic transmission values in which the corresponding absorption will reach plateau values, i.e., the absorptance threshold value, given by $A_{\max }(\lambda)$. Eight layers of the white textile (almost $100 \mathrm{mg} / \mathrm{cm}^{2}$ ) transmitted around $3 \%$ of the incoming light [violet starts, Fig. 7(b)]. For more layers, we speculate that the absorptance of the white textile would plateau like the other samples. That is because more layers of the white textile are necessary to sufficiently decrease the transmission through the textile stack. (For more details, we refer to the discussion in Sec. 5.4.)

\subsection{SWIR: $1450 \mathrm{~nm}$}

We also fitted the experimental data with the extinction model for incoming light with a wavelength of $1450 \mathrm{~nm}$ (Fig. 8). The fit is good for all data, except some small deviations observed for the smallest transmission data (high mass per area) and the absorptance data of the first layer of the samples. Due to the absorptance band of water at $1450 \mathrm{~nm}$, the moist birch leaf transmits little light $(<10 \%)$ and varies little with mass per area, i.e., with the number of leaf layers [red squares, Fig. 8(a)]. The textiles reflected more light and reached a reflectance plateau around $70 \mathrm{mg} / \mathrm{cm}^{2}$ for the white and P1 textile, and around $40 \mathrm{mg} / \mathrm{cm}^{2}$ for the P2 textile [Fig. 8(a)].

The decreased transmission of the samples versus an increase in mass per area [Fig. 8(b)] was similar for the samples (extinction coefficients 0.03 to 0.06 ). The transmission data from the birch leaves and the P2 textile are partly overlapping, both with an extinction coefficient equal to 0.06 . At $50 \mathrm{mg} / \mathrm{cm}^{2}$, corresponding to slightly more than two birch leaf layers, the transmission curves of the white textile overlaps with that of the birch leaf and P2 textile [Fig. 8(b)].

At $1450 \mathrm{~nm}$, the absorptance of the samples reaches a plateau around $90 \mathrm{mg} / \mathrm{cm}^{2}$ for the leaves and P2 textile, and around $120 \mathrm{mg} / \mathrm{cm}^{2}$ for the P1 textile. As observed at 700 and $1000 \mathrm{~nm}$, the white textile did not reach an absorptance plateau below $90 \mathrm{mg} / \mathrm{cm}^{2}$ at $1450 \mathrm{~nm}$ (corresponding to 8 layers). The transmission through a white textile stack of $90 \mathrm{mg} / \mathrm{cm}^{2}$ is close to $2 \%$. More layers of the textile are therefore necessary to reach an absorptance plateau, which we speculate would occur at mass per area values above $150 \mathrm{mg} / \mathrm{cm}^{2}$. This value is expected for higher values than the P1 textile because the P1 textile had a slightly higher extinction coefficient of the two materials. 
Mikkelsen and Selj: Spectral characteristics of moist birch leaves and synthetic materials...

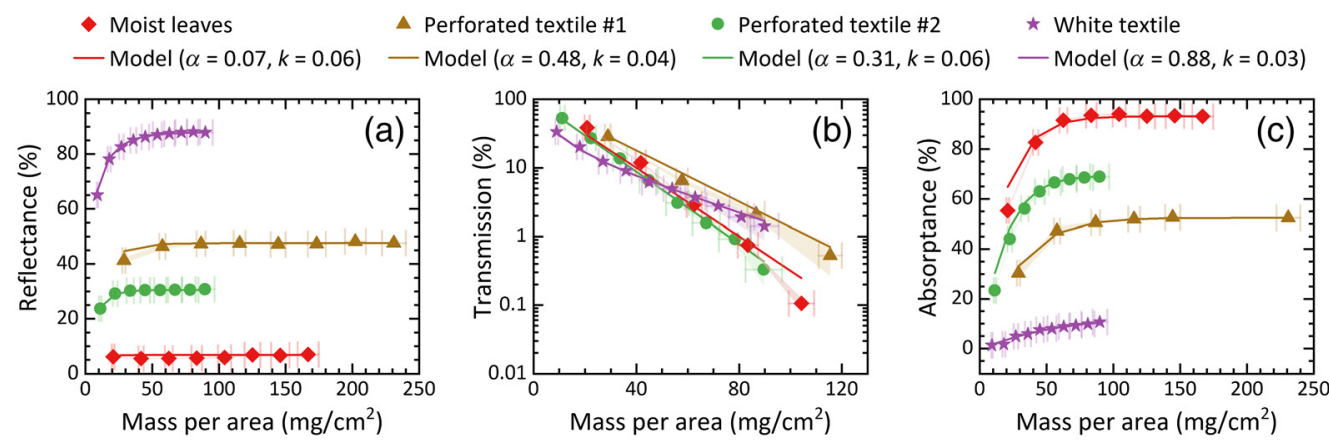

Fig. 8 Spectral properties versus mass per area for moist birch leaves (red squares), perforated textile \#1 (sand triangles), perforated textile \#2 (green circles), and white textile (violet stars) samples at $1450 \mathrm{~nm}$ : measured (a) reflectance, (b) transmission, and (c) absorptance of the samples (mean values) plotted against mass per area. The data points are fitted to the extinction model for each sample (solid lines) with different reflectance coefficients $(\boldsymbol{\alpha})$ and extinction coefficients $(k)$ with dimension $\mathrm{cm}^{2} / \mathrm{mg}$. The semitransparent areas around the curves represent SEM values of the data (errors smaller than the lines might not be visible in the plot), while the horizontal error bars represent the error of the samples' mass per area.

Overall, we find that the P2 textile best resembled the spectral characteristics of the moist birch leaf at $1450 \mathrm{~nm}$. The P2 textile reflected slightly more of the lights than the leaf but the transmission of light through multiple layers of the textile resembles that of the leaves for similar mass per area values.

\subsection{Result Trends and Understanding the Model Parameters}

From the results presented in Sec. 5.1, we found some common trends among the results (at the three given wavelengths) that we will discuss here.

Samples that had the same extinction coefficient $(k)$ seem to reach an absorption plateau around the same mass per area value, i.e., they have the same absorption threshold value $A_{\max }(\lambda)$ for a given wavelength. For instance, the moist leaves and the P1 textile both have $k=$ 0.05 at $\lambda=700 \mathrm{~nm}$. Both these samples reach an absorption plateau around $90 \mathrm{mg} / \mathrm{cm}^{2}$ [Fig. 6(c)]. At the same mass per area value, these two samples had a transmission value close to $1 \%$ [Fig. 6(b)]. For the three wavelengths studied in this paper, we found that the samples reached an absorption plateau when their transmission was $\sim 1 \%$, which seems to be a transmission threshold level. Overall, we observed that samples with a large extinction coefficient absorbed more light compared to samples with a lower extinction coefficient [Figs. 2 and 5(c)]. The transmission of samples having a high $k$ value thus decreases fast toward zero when their mass per area (or the number of layers) increases.

Some of the investigated samples did not have a mass per area value large enough for them to reach the absorption plateau, i.e., the mass per area was smaller than their absorption threshold values. Using the extinction model on the transmission data, it is possible to extrapolate the data to estimate in which mass per area values the transmission is expected to reach $1 \%$ levels and thus the sample's $A_{\max }(\lambda)$ value. As an example, we estimated the absorption threshold value of the white textile [from the fit in Fig. 6(b), violet line] to be around $150 \mathrm{mg} / \mathrm{cm}^{2}$ at $700 \mathrm{~nm}$.

In addition to the extinction coefficient (that accounts for sample absorption), the extinction model also uses the reflectivity $(\alpha)$ of the sample as a fitting parameter. The reflectivity, which is a property of the material, must not be confused with the sample reflectance, a value that changes with the thickness of the sample. When the sample is homogeneous and sufficiently thick, the sample's reflectance and reflectivity values coincide. In other words, when the reflectivity of a sample is known or estimated (e.g., from a model), one then knows the maximum reflectance of the sample (that it will converge to) when its thickness or number of layers approaches infinity. To resemble the reflectance of a natural biomaterial sample (e.g., a leaf), it is not always sufficient for the synthetic sample to match the reflectivity of the biomaterial. Even if their reflectivity values are similar, the samples can have different reflectance values when the sample is thin, 
perforated, or have too few layers due to different transmission and absorption values (related to the extinction coefficient). For many practical purposes such as remote sensing applications and camouflage, sensors capture the reflectance and not necessarily reflectivity values (of any two different objects, natural or synthetic).

In our studies, none of the textiles matched the reflectivity of the moist leaves at the chosen wavelengths $(700,1000$, and $1450 \mathrm{~nm})$. For all the textiles and studied wavelengths, the P2 textile was closest at matching the reflectivity of the leaf at $1000 \mathrm{~nm}$ with $\alpha_{\mathrm{P} 2}(1000)=0.27$ compared to the reflectivity of the leaf $\alpha_{L}(1000)=0.32$ [Fig. 7(a)]. Due to differences in transmission and absorption properties between the samples, their reflectance values overlapped at $40 \mathrm{mg} / \mathrm{cm}^{2}$ (corresponding to almost two leaf layers and four textile layers). This shows that even if the reflectivity values of two samples are different, their reflectance can still overlap at some thickness (mass per area or layers) if the sample with the lowest reflectivity also has lower transmission and/or extinction coefficient than the other sample.

Biomaterials are very diverse regarding their spectral properties. The properties of a biomaterial sample, e.g., a specific leaf, might change due to factor such as environment and climate variations, water content, diseases, and leaf thickness. To mimic biomaterial samples, it is therefore important to tailor the spectral properties of the synthetic material to fit the typical reflectance and transmission values of the biomaterial expected at a certain scene. For instance, the effective thickness of a leaf canopy can vary greatly depending on the location and time of year. Having a camouflage material that resembles the spectral properties of the scene for different thicknesses will thus be a valuable advantage.

\section{Two-Way Transmission: Model Comparison}

To compare the different models presented in Sec. 3, we calculated the two-way transmission of the different samples using the models and spectral measurements. For the Lillesaeter model, we calculated the two-way transmission [using Eq. (11)] and the following measurements: (1) reflectance of the black background $\left(\beta_{D}\right)$; (2) reflectance of the white background $\left(\beta_{L}\right) ;(3)$ reflectance of a single layer of each of the samples on the black background $\left(r_{1 D}\right)$; and (4) reflectance of a single layer of each of the samples on a white background $\left(r_{1 L}\right)$. The multiple-scattering model [Eq. (17)] required the same measurement inputs as the Lillesaeter model.

The extinction model [Eqs. (5)-(7)] can be fitted to reflectance, transmission, and absorptance measurements of a sample plotted against the number of layers (or other related properties, e.g., mass per area or thickness) at a chosen wavelength. If the samples are placed on a background, the reflectance of the background must also be measured. To check the accuracy of the two-way transmission calculated from the extinction model (compared to experimental data and the other two models), we fitted the model to the reflectance and transmission measurements of the samples (on a black background) for 1 to 8 layers at selected wavelengths: 400, 700, 1000, 1300, 1450, 1850, and $2200 \mathrm{~nm}$. One of the fitting parameters, the reflectivity of the samples, was set equal to the measured reflectance of 6 to 8 layers of each sample. After fitting the model to the data (choosing the best extinction coefficient), we used the fitting parameters (sample reflectivity and extinction coefficient) to calculate the transmission of each sample and thus the two-way transmission.

Figure 9 presents the estimated two-way transmission of each sample using the different models. Their values are compared with the measured two-way transmission (multiplication of the transmission through each sample when faced forward and backward). The multiplescattering model fits best with the birch leaf measurements [Fig. 9(a)], especially for wavelengths shorter than $1400 \mathrm{~nm}$. At longer wavelengths, all three models slightly underestimate the two-way transmission. The extinction model underestimates the transmission for all wavelengths except UV and VIS, whereas the Lillesaeter model overestimates the transmission between 850 and $1350 \mathrm{~nm}$. At these wavelengths, the absorptance of the birch leaf was lowest [Fig. 2(c)], i.e., the sum of the light that was reflected and transmitted was highest. When the reflectance and transmittance of a sample are high, more light from multiple scattering between the sample and background is reflected back to the sensor. The Lillesaeter model does not account for multiple scattering. Consequently, it underestimates the reflectance of the system (sample + background) and overestimates the transmission. 


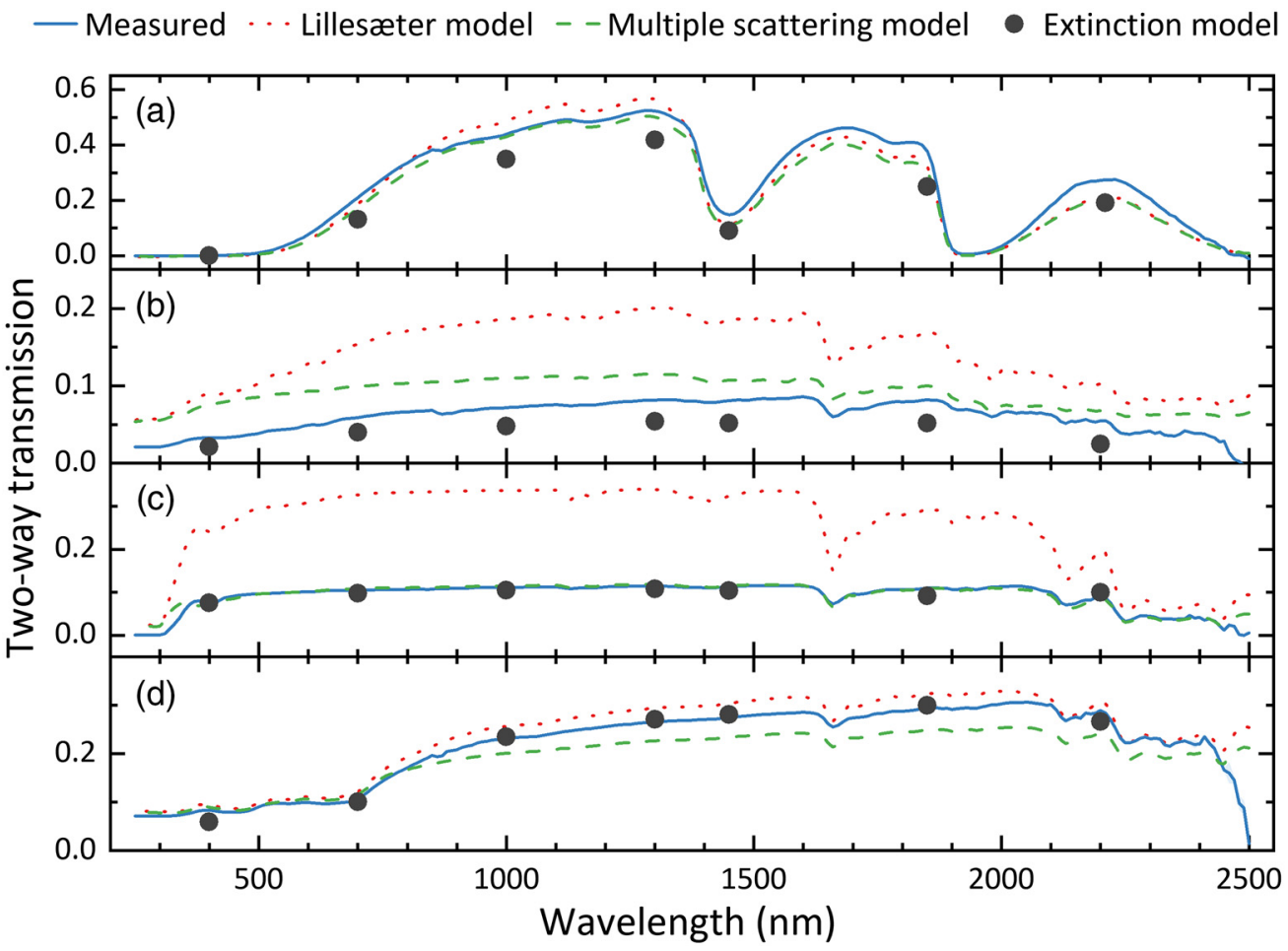

Fig. 9 Two-way transmission versus wavelength for: (a) moist leaf, (b) perforated textile \#1, (c) perforated textile \#2, and (d) white textile. The measured transmission (mean values, solid blue lines) is compared with three models: Lillesaeter's model (red dotted lines), multiple scattering model (green dashed lines), and the extinction model (black circles). The semitransparent areas around the curves represent SEM values of the data (errors smaller than the lines might not be visible in the plot).

For the P1 textile [Fig. 9(b)], both the Lillesaeter and multiple scattering models overestimate the two-way transmission over all wavelengths, whereas the extinction model best fits the data (although it slightly underestimates the transmission). The reflectance of the P1 textile [Fig. 4(a)] is for most of the wavelengths, more than twice that of the birch leaf [Fig. 2(a)]. This is an important difference for the light attenuation induced by multiple scattering, i.e., the light intensity is reduced for each time light scatters between the sample and the background, given by their multiplied reflectivity: $r \beta$. The Lillesaeter model does not capture these scattering effects, hence the large difference we observe between the two-way transmission values of this model and the multiple-scattering model. The multiple-scattering model likely overestimates the transmission through the P1 textile because the textile has a high absorptance [Fig. 4(c)], whereas absorptance is not included in the model itself.

The Lillesaeter model fits better to the measured two-way transmission data of the P2 textile [Fig. 9(c)] than to the P1 textile. We believe that is because the P2 textile reflects less light than the P1 textile. Considering the P1 textile, the model only overestimates the data between 700 and $2100 \mathrm{~nm}$. That is where the reflectance [Fig. 5(a)] and transmission values were highest [Fig. 5(b)]. For wavelengths longer than $800 \mathrm{~nm}$, the multiple-scattering model underestimates the two-way transmission [green dashed line, Fig. 9(c)], however, not by more than 20\%. The extinction model overlaps the data points for all wavelengths and is thus the model that best fits the two-way transmission data of the P2 textile.

The white textile has the highest reflectance [Fig. 3(a)] of all the samples. As expected, due to the sample's high reflectance and multiple scattering effects, the estimated two-way transmission is much higher for the Lillesaeter model compared to the other models and the measurements [Fig. 9(d)]. Both the multiple-scattering model and extinction model fit well with the two-way transmission data. 
To summarize, we find that the Lillesaeter model, which is the simplest of the three models, deviates most from the measurements. Because it does not account for inevitable multiple scattering between the sample and background, it should not be used for measurements on samples placed on a high-reflecting background, especially not if the sample also has a high reflectance, as seen for the white textile in our study. The multiple-scattering model is, as we would expect, more accurate in estimating the two-way transmission of the samples, as it also captures more of the relevant scattering physics of thin, multi-layered samples. To avoid significant errors, we therefore recommend using the multiple-scattering model over the Lillesaeter model. Both models are simple and time-effective, and only require four types of reflectance measurements: measurements of two different backgrounds and one reflectance measurement of a single layer of the specific sample on each of the two backgrounds. However, the two models are hampered by the fact that they do not account for sample and background absorptance. When the two-way transmission of the sample is estimated, it is easy to calculate the reflectance or transmission of multiple-layers of the sample.

The extinction model is more advanced and realistic as it accounts for absorption, multiple scattering, and characteristics of the background. It requires more measurements than the other two models, e.g., reflectance measurements of the background and multiple layers of the sample. The reflectance measurements can be supplemented (to improve the accuracy of the model) or substituted by transmission and/or absorptance measurements (e.g., if that is more practical). For the samples tested here, we found that the extinction model often underestimates the transmission [Figs. 2 and 5(b)] and overestimates the reflectance [Figs. 2 and 5(a)] of a single layer of the samples. However, the difference is small, and the model fits well with the reflectance and transmission data for multiple layers of the samples.

\section{Conclusions}

In this study, we measured and compared the spectral characteristics (between 250 and $2500 \mathrm{~nm}$ ) of samples of 1 to 8 layers of brown, moist birch leaves with three different synthetic materials relevant for camouflage purposes. Birch leaves are interesting from an optical point of view as they are found throughout Northern Europe in several months of each year. Our results show that the birch leaves transmitted light, in the NIR and part of the SWIR region, up to 7 to 8 layers thick. For other wavelengths, in the visible range, the leaves were less transparent and only 2 to 3 layers were then required to prevent reflection effects from a black background. On the other hand, and depending on the wavelength, the thickest of the synthetic materials (perforated textiles P1 and P2) required 2 to 4 layers, whereas the thinnest materials required 5 to 6 layers to hinder reflectance contributions from the background.

To compare the spectral properties of the different samples of biomaterials and synthetic materials, we measured their mass per area and tested their reflectance, transmission, and absorptance data against an extinction model. The model uses the reflectance of the background, the spectral data of each sample measured for multiple layers, and two fitting parameters: the sample's reflectivity and extinction coefficient (a high extinction coefficient value implies that the sample absorptance is large per mass per unit area, i.e., the incident light is absorbed more rapid for a given sample thickness). The model fitted very well with the spectral data. We only observed some minor deviations from the data at very small transmission values $(<1 \%)$ and for the reflectance data for some of the single-layered samples. The former is probably due to accuracy limitations in the transmission measurements.

The extinction model estimates the reflectance, transmission, and absorptance of a sample based on multi-layered measurements of at least one of these three spectral properties. This is especially valuable if it is not possible to measure the remaining two properties, e.g., due to time limitations, lack of resources, or for samples not fitting well into lab measurement setups. Using the model, one may therefore overcome challenges induced by temperature (snow and ice samples) and sample material brittleness (granular materials and similar). With a sufficient number of data points, the model also estimates the spectral properties of a sample versus its thickness (i.e., number of layers). By adjusting the thickness of camouflage material, it is possible to match the spectral properties of a biomaterial of a certain mass per area. For example, at $700 \mathrm{~nm}$, we 
measured that a stack of birch leaves and a stack of P2 textile layers had the same reflectance when their mass per area was $\sim 40 \mathrm{mg} / \mathrm{cm}^{2}$ (corresponding to approximately two birch leaf layers and almost four P2 textile layers). Moreover, we discussed (Sec. 5) how the extinction model can estimate the mass per area when the white textile approach an absorption (and reflectance) plateau. Overall, we observed that all samples approached an absorption plateau when their transmission was less than approximately $1 \%$. The samples with the highest extinction coefficient absorbed more of the incoming light and their corresponding transmission decreased fastest when the mass per area was increased, as expected.

To resemble spectral characteristics of biomaterials at various optical depths, synthetic materials, such as those relevant for camouflage purposes, should match both the wavelengthdependent extinction coefficient and reflectivity of the biomaterial. Similar reflectivity ensures that different materials will have the same reflectance when they are homogeneous and sufficiently thick. If the materials also have the same extinction coefficient, their transmission versus thickness will be similar, ensuring small deviations between their reflectance and absorptance values as their thickness varies.

We also compared the extinction model with two simpler reflectance models that do not account for absorption effects and therefore require fewer measurements as input: (i) a model proposed by Lillesaeter ${ }^{30}$ that requires reflectance measurements of the samples at two different backgrounds and (ii) a modified version of the Lillesaeter model that accounts for multiple scattering effects. In a previous work, we have used the former model to estimate the reflectance of multi-layered camouflage nets. ${ }^{17}$ To compare the models, we now calculated the two-way transmission of the different samples by utilizing each model. We then plotted the results together with the measured data. Overall, the extinction model had the smallest deviation from the transmission data. The multiple scattering models also fitted the data reasonably well, whereas the Lillesaeter model often overestimated the transmission, especially when the samples had high reflectance values. Because the Lillesaeter model does not account for multiple-scattering effects, we believe that it should not be used on samples with high reflectance and transparency, especially not when placed on backgrounds with high reflectance values.

We expect that our findings and discussions will be useful for the development of synthetic materials made to mimic natural biomaterials, such as materials for camouflage purposes. The results highlight the importance of choosing appropriate material thickness when designing camouflage. Furthermore, the results are considered to be of importance for wavelength-dependent detections based on spectral anomalies of artificial objects when sensed through vegetation layers. The results should also be relevant for advanced multilayered fabric technology and remote sensing applications where detailed knowledge on the effect of canopy backgrounds on the data captured is vital for precise land mapping. ${ }^{29,54,55}$ Future studies should investigate further how the spectral characteristics of textiles change with textile densities and weaving. This would be useful for choosing textile material properties that will best match the spectral behavior of biomaterial. In addition, the extinction model should be further tested. It would also be valuable to study the spectral properties of green leaves, leaves versus water content, and thickness, and compare those to camouflage material. Finally, to expand the utility of the extinction model, extended full spectrum should be found, covering all measured wavelengths.

\section{Acknowledgments}

The work presented here was funded by Norwegian Defence and Research Establishment. The authors have no conflicts of interests.

\section{References}

1. J. A. Endler, "Disruptive and cryptic coloration," Proc. Biol. Sci. 273, 2425-2426 (2006).

2. O. Lillesaeter, "Complex contrast, a definition for structured targets and backgrounds," J. Opt. Soc. Am. A 10, 2453-2457 (1993).

3. F. Racek et al., "Selected issues connected with determination of requirements of spectral properties of camouflage patterns," Proc. SPIE 10432, 1043205 (2017). 
Mikkelsen and Selj: Spectral characteristics of moist birch leaves and synthetic materials...

4. A. Zavvartorbati, H. Dehghani, and A. J. Rashidi, "Evaluation of camouflage effectiveness using hyperspectral images (Erratum)," J. Appl. Remote Sens. 12, 019901 (2018).

5. C. Birkemark, "CAMEVA, a methodology for estimation of target detectability," Opt. Eng. 40 1835-1843 (2001).

6. G. Selj, "Disruptive camouflage tricks the human eye: a study of detection times of two near-similar targets in natural backgrounds," Proc. SPIE 9653, 96530S (2015).

7. I. C. Cuthill et al., "Disruptive coloration and background pattern matching," Nature 434, 72-74 (2005).

8. A. Toet and M. Hogervorst, "Urban camouflage assessment through visual search and computational saliency," Opt. Eng. 52, 041103 (2012).

9. D. Heinrich and G. Selj, "The effect of contrast in camouflage patterns on detectability by human observers and CAMAELEON," Proc. SPIE 9476, 947604 (2015).

10. A. Schwegmann, "Camouflage evaluation by bio-inspired local conspicuity quantification," Proc. SPIE 10794, 107940H (2018).

11. J. Jersblad and P. Jacobs, "Thermal transmission of camouflage nets revisited," Proc. SPIE 9997, 99970S (2016).

12. J. Jersblad and C. Larsson, "Camouflage effectiveness of static nets in SAR images," Proc. SPIE 9653, 965304 (2015).

13. G. Selj and D. Heinrich, "A field-based method for evaluating thermal properties of static and mobile camouflage," Proc. SPIE 10794, 107940B (2018).

14. O. Dev et al., "Multi-layered textile structure for thermal signature suppression of ground based targets," Infrared Phys. Technol. 105, 103175 (2020).

15. X. Yin, Q. Chen, and N. Pan, "A study and a design criterion for multilayer-structure in perspiration based infrared camouflage," Exp. Therm. Fluid Sci. 46, 211-220 (2013).

16. S. Brzeziński et al., "Textile multi-layer systems for protection against electromagnetic radiation," Fibres Text. East. Eur. 17, 66-71 (2009).

17. A. Mikkelsen and G. Selj, "Spectral reflectance and transmission properties of a multilayered camouflage net: comparison with natural birch leaves and mathematical models," Proc. SPIE 11536, 1153609 (2020).

18. J. Loyd and J. Sanders, "Physically realistic camouflage net models for visualization and signature generation," Proc. SPIE 4370, 72-83 (2001).

19. H. Dong et al., "Propagation characteristic of THz wave in camouflage net material," Proc. SPIE 10461, 104611J (2017).

20. G. Cui et al., "Analysis and research on thermal infrared properties and adaptability of the camouflage net," Proc. SPIE 10157, 1015735 (2016).

21. D. Heinrich and G. Selj, "Evaluation of camouflage pattern performance of textiles by human observers and CAMAELEON," Proc. SPIE 10432, 1043206 (2017).

22. G. Selj and D. Heinrich, "Search by photo methodology for signature properties assessment by human observers," Proc. SPIE 9474, 947411 (2015).

23. M. Hogervorst, A. Toet, and P. Jacobs, "Design and evaluation of (urban) camouflage," Proc. SPIE 7662, 766205 (2010).

24. A. Toet and M. Hoogervorst, "Review of camouflage assessment techniques," Proc. SPIE 11536, 1153604 (2019).

25. R. H. Wilhelm and J. B. Smith, "Transmittance, reflectance, and absorptance of near infrared radiation in textile materials," Text. Res. J. 19, 73-88 (1949).

26. J. M. Preston and P. C. Tsien, "The cellulose-dyestuff complex. Part II: The intensity of light reflected from dyed fibres*," J. Soc. Dye. Colour. 62, 242-248 (1946).

27. E. I. Stearns, "Selected ordinate method for interpretation of spectrophotometric data," Text. Res. 14, 326-332 (1944).

28. H. Zhang, T. Hu, and J. Zhang, "Transmittance of infrared radiation through fabric in the range $8-14 \mu \mathrm{m}$," Text. Res. J. 80, 1516-1521 (2010).

29. G. M. Berard, E. A. Cloutis, and P. Mann, "Leaf reflectance and transmission properties (350-2500 nm): implications for vegetation indices," J. Near Infrared Spectrosc. 25, $138-144$ (2017).

30. O. Lillesaeter, "Spectral reflectance of partly transmitting leaves: laboratory measurements and mathematical modeling," Remote Sens. Environ. 12, 247-254 (1982). 
31. W. A. Allen and A. J. Richardson, "Interaction of light with a plant canopy," J. Opt. Soc. Am. 58, 1023-1028 (1968).

32. A. A. Kokhanovsky, "Physical interpretation and accuracy of the Kubelka-Munk theory," J. Phys. D Appl. Phys. 40, 2210-2216 (2007).

33. S. L. Ustin, S. Jacquemoud, and Y. Govaerts, "Simulation of photon transport in a threedimensional leaf: implications for photosynthesis," Plant Cell Environ. 24, 1095-1103 (2001).

34. P. Zarco-Tejada, "Development of a vegetation fluorescence canopy model," ESA Scientific and Technical Publications Branch, ESTEC (2005).

35. W. Verhoef, "Simultaneous retrieval of soil, leaf, canopy, and atmospheric parameters from hyperspectral information in the red edge through model inversion," Proc. SPIE 3868, 380-387 (1999).

36. G. Melillos et al., "Detecting underground structures in Cyprus using field spectroscopy," Proc. SPIE 10773, 107730A (2018).

37. Q. Jiao et al., "Study on the predicted model of crop leaf water status by the NIR band of ground reflectance and spaceborne hyperspectral images," Proc. SPIE 6835, 68351H (2008).

38. D. Rosario and A. Ortiz, "Spectral-elevation data registration using visible-SWIR spatial correspondence," Proc. SPIE 10644, 106440C (2018).

39. P. Coppo, L. Chiarantini, and L. Alparone, "Design and validation of an end-to-end simulator for imaging spectrometers," Opt. Eng. 51, 111721 (2012).

40. L. Grant, "Diffuse and specular characteristics of leaf reflectance," Remote Sens. Environ. 22, 309-322 (1987).

41. S. Matthews, "Effect of drying temperature on fuel moisture content measurements," Int. J. Wildland Fire 19, 800-802 (2010).

42. D. M. Gates et al., "Spectral properties of plants," Appl. Opt. 4, 11-20 (1965).

43. K. F. Palmer and D. Williams, "Optical properties of water in the near infrared*," J. Opt. Soc. Am. 64, 1107-1110 (1974).

44. S. Jacquemoud and S. Ustin, Leaf Optical Properties, Cambridge University Press (2019).

45. H. Mattila et al., "Degradation of chlorophyll and synthesis of flavonols during autumn senescence - the story told by individual leaves," AoB Plants 10, ply028 (2018).

46. O. Virtanen, E. Constantinidou, and E. Tyystjärvi, "Chlorophyll does not reflect green light: how to correct a misconception," J. Biol. Educ. 1-8 (2020).

47. S. V. Ollinger, "Sources of variability in canopy reflectance and the convergent properties of plants," New Phytol. 189, 375-394 (2011).

48. A. Hovi, P. Raitio, and M. Rautiainen, "A spectral analysis of 25 boreal tree species," Silva Fenn 51, 1-16 (2017).

49. A. D. Richardson and G. P. Berlyn, "Changes in foliar spectral reflectance and chlorophyll fluorescence of four temperate species following branch cutting," Tree Physiol. 22, 499-506 (2002).

50. S. Foley et al., "Foliar spectral properties following leaf clipping and implications for handling techniques," Remote Sens. Environ. 103, 265-275 (2006).

51. W. Yamori, "Photosynthesis and respiration," Chapter 12 in Plant Factory, T. Kozai, G. Niu, and M. Takagaki, Eds., 2nd ed., pp. 197-206, Academic Press (2020).

52. P. Ceccato et al., "Detecting vegetation leaf water content using reflectance in the optical domain," Remote Sens. Environ. 77, 22-33 (2001).

53. R. Colombo et al., "Estimation of leaf and canopy water content in poplar plantations by means of hyperspectral indices and inverse modeling," Remote Sens. Environ. 112, 1820-1834 (2008).

54. S. Jacquemoud et al., "PROSPECT + SAIL models: a review of use for vegetation characterization," Remote Sens. Environ. 113, S56-S66 (2009).

55. L. Liu, S. Zhang, and B. Zhang, "Evaluation of hyperspectral indices for retrieval of c anopy equivalent water thickness and gravimetric water content," Int. J. Remote Sens. 37, 3384-3399 (2016). 
Alexander Mikkelsen received his $\mathrm{MSc}$ and $\mathrm{PhD}$ degrees in physics from Norwegian University of Science and Technology in 2010 and 2013, respectively. He is a scientist at Norwegian Defence Research Establishment. His background is from soft matter physics, researching emulsion droplets, and self-assembly of nano-micrometer particles. He is the author of more than 20 journal papers. His current research interests include camouflage and signature management, camouflage testing and assessment, and concealment technologies.

Gorm K. Selj received his MSc and PhD degrees in physics from the University of Oslo in 2006 and 2010, respectively. He is a senior scientist at Norwegian Defence Research Establishment. His background is from theoretical physics, bioimpedance, as well as complex matter physics. His current research interests include camouflage technologies and signature management. 